\title{
Morris Water Maze (MWM)
}

\section{Study: Pregabalin \& L-Theanine Prophylactic Effects on PTSD Behavior and Gene Expression in Male Sprague-Dawley Rats}

Per the protocol the aims of this study are as follows (amended 10/13/17):

\section{SPECIFIC AIMS}

The aims of these studies are to determine if preemptive administration of PGB or L-Th prevent PTSD development in the rodent model. Specifically, the aims are as follows:

1. Determine the effects of PGB and L-Th on anxiety

2. Determine the effects of PGB and L-Th on locomotion

3. Determine the effects of PGB and L-Th on memory

4. Determine the effects of PGB and L-Th on depression

5. Determine the effects of PGB and L-Th on gene expression in the brain (p. 14).

And:

The aims of this research protocol will be guided by the following questions:

1. Is there a significant difference in the anxiolytic effects between the groups?

2. Is there a significant difference in locomotion between the groups?

3. Is there a significant difference in memory between the groups?

4. Is there a significant difference in depression between the groups?

5. Are there significant differences in gene expression and regulation in the hippocampus between the groups?

6. Are there significant differences in gene expression and regulation in the amygdala $b$ etween the groups? (p. 15). 
The grouping variable is as follows:

There was a total of 6 groups (1-6), each with 10 rat subjects.

The three groups of non-stressed rats:

1 - control-vehicle (received vehicle injections BID);

2 - L-Th control drug (received PGB BID)*

3 - control-naïve (received no injections)

The three groups in the 3-day restraint/shock stressed rats:

4 - PTSD-vehicle (received vehicle injection BID after three-day restraint/shock);

5 - PTSD-drug pre-treatment (received PGB BID 24 hours before and for a period of 10 days after three-day restraint shock );

6 - PTSD-post-treatment (received PGB injections BID for 10 days after three-day restraint/shock)

\begin{tabular}{|c|c|c|c|c|c|}
\hline \multicolumn{6}{|c|}{ Group } \\
\hline & & Frequency & Percent & Valid Percent & $\begin{array}{c}\text { Cumulative } \\
\text { Percent }\end{array}$ \\
\hline \multirow[t]{7}{*}{ Valid } & 1 control-vehicle & 10 & 16.7 & 16.7 & 16.7 \\
\hline & $2 \mathrm{~L}-\mathrm{Th}$ control drug & 10 & 16.7 & 16.7 & 33.3 \\
\hline & 3 control-naïve & 10 & 16.7 & 16.7 & 50.0 \\
\hline & 4 PTSD-vehicle & 10 & 16.7 & 16.7 & 66.7 \\
\hline & $\begin{array}{l}5 \text { PTSD-drug pre- } \\
\text { treatment }\end{array}$ & 10 & 16.7 & 16.7 & 83.3 \\
\hline & 6 PTSD-post-treatment & 10 & 16.7 & 16.7 & 100.0 \\
\hline & Total & 60 & 100.0 & 100.0 & \\
\hline
\end{tabular}

Statistical Analysis: For this design a one-way ANOVA will be conducted for each of the outcome variables. All assumptions will be examined including homogeneity of error variances (via the Levine test) and normality. The eta-squared $\left(\eta^{2}\right)$ effect size will be reported. Though interpreting and casting judgment as to what constitutes a small/medium/large effect size is context-dependent using Cohen's (1988) taxonomy .01/.059/138 will be small/medium/large. As well, all outliers and data anomalies will be examined and addressed accordingly (e.g., transformations, nonparametric options, etc.). In the event of a significant result $(\alpha=.05)$ post hoc tests (e.g., Tukey's HSD) will be performed. Descriptive statistics and graphics will be provided for the full sample $(n=60)$ and by group.

Cohen (1988). Statistical power analysis for the behavioral sciences. (2nd Ed.). Hillsdale, NJ: Lawrence Erlbaum. 


\section{Descriptive Statistics: Full sample}

\begin{tabular}{|c|c|c|c|c|c|c|c|c|c|}
\hline \multicolumn{10}{|c|}{ Statistics } \\
\hline & & Distance & $\begin{array}{l}\text { Meanspeed } \\
\text { Mean speed }\end{array}$ & $\begin{array}{l}\text { Maxspeed } \\
\text { Max speed }\end{array}$ & $\begin{array}{l}\text { ZONE1 entrie } \\
\text { s ZONE 1: } \\
\text { entries }\end{array}$ & $\begin{array}{l}\text { ZONE1 time } \\
\text { ZONE } 1 \text { : time }\end{array}$ & $\begin{array}{l}\text { ZONE1 distan } \\
\text { ce ZONE 1: } \\
\text { distance }\end{array}$ & $\begin{array}{l}\text { ZONE1 latenc } \\
\text { ytofirstentry } \\
\text { ZONE 1: } \\
\text { latency to first } \\
\text { entry }\end{array}$ & $\begin{array}{l}\text { ZONE1 maxsp } \\
\text { eed ZONE } 1 \text { : } \\
\text { max speed }\end{array}$ \\
\hline \multirow[t]{2}{*}{$\mathrm{N}$} & Valid & 60 & 60 & 60 & 60 & 60 & 60 & 60 & 60 \\
\hline & Missing & 0 & 0 & 0 & 0 & 0 & 0 & 0 & 0 \\
\hline \multicolumn{2}{|c|}{ Mean } & 14.75612 & .24592 & .40417 & 5.22 & 16.943 & 4.05363 & 7.200 & .40927 \\
\hline \multicolumn{2}{|c|}{ Std. Error of Mean } & .211584 & .003525 & .004257 & .215 & .7796 & .160388 & .8616 & .006544 \\
\hline \multicolumn{2}{|c|}{ Median } & 14.87250 & .24800 & .40700 & 5.00 & 16.950 & 4.09200 & 5.800 & .40650 \\
\hline \multicolumn{2}{|c|}{ Mode } & 16.745 & .248 & .428 & 5 & $10.5^{\mathrm{a}}$ & $4.233^{\mathrm{a}}$ & $.0^{\mathrm{a}}$ & .398 \\
\hline \multicolumn{2}{|c|}{ Std. Deviation } & 1.638925 & .027303 & .032974 & 1.668 & 6.0388 & 1.242361 & 6.6741 & .050690 \\
\hline \multicolumn{2}{|c|}{ Variance } & 2.686 & .001 & .001 & 2.783 & 36.467 & 1.543 & 44.543 & .003 \\
\hline \multicolumn{2}{|c|}{ Skewness } & -.051 & -.047 & -1.022 & .437 & .655 & .337 & 1.340 & 1.223 \\
\hline \multicolumn{2}{|c|}{ Std. Error of Skewness } & .309 & .309 & .309 & .309 & .309 & .309 & .309 & .309 \\
\hline \multicolumn{2}{|c|}{ Kurtosis } & -.721 & -.712 & 3.479 & -.114 & .788 & .230 & 1.827 & 5.691 \\
\hline \multicolumn{2}{|c|}{ Std. Error of Kurtosis } & .608 & .608 & .608 & .608 & .608 & .608 & .608 & .608 \\
\hline \multicolumn{2}{|c|}{ Range } & 6.627 & .111 & .200 & 8 & 30.7 & 6.330 & 28.8 & .354 \\
\hline \multicolumn{2}{|c|}{ Minimum } & 11.347 & .189 & .270 & 2 & 4.3 & .999 & .0 & .277 \\
\hline \multicolumn{2}{|c|}{ Maximum } & 17.974 & .300 & .470 & 10 & 35.0 & 7.329 & 28.8 & .631 \\
\hline \multicolumn{2}{|c|}{ Sum } & 885.367 & 14.755 & 24.250 & 313 & 1016.6 & 243.218 & 432.0 & 24.556 \\
\hline
\end{tabular}

a. Multiple modes exist. The smallest value is shown 
Distance

\begin{tabular}{|c|c|c|c|c|c|}
\hline & & Frequency & Percent & Valid Percent & $\begin{array}{c}\text { Cumulative } \\
\text { Percent }\end{array}$ \\
\hline \multirow[t]{59}{*}{ Valid } & 11.347 & 1 & 1.7 & 1.7 & 1.7 \\
\hline & 11.778 & 1 & 1.7 & 1.7 & 3.3 \\
\hline & 11.972 & 1 & 1.7 & 1.7 & 5.0 \\
\hline & 12.092 & 1 & 1.7 & 1.7 & 6.7 \\
\hline & 12.262 & 1 & 1.7 & 1.7 & 8.3 \\
\hline & 12.413 & 1 & 1.7 & 1.7 & 10.0 \\
\hline & 12.588 & 1 & 1.7 & 1.7 & 11.7 \\
\hline & 12.752 & 1 & 1.7 & 1.7 & 13.3 \\
\hline & 12.800 & 1 & 1.7 & 1.7 & 15.0 \\
\hline & 12.936 & 1 & 1.7 & 1.7 & 16.7 \\
\hline & 12.978 & 1 & 1.7 & 1.7 & 18.3 \\
\hline & 13.102 & 1 & 1.7 & 1.7 & 20.0 \\
\hline & 13.105 & 1 & 1.7 & 1.7 & 21.7 \\
\hline & 13.367 & 1 & 1.7 & 1.7 & 23.3 \\
\hline & 13.585 & 1 & 1.7 & 1.7 & 25.0 \\
\hline & 13.667 & 1 & 1.7 & 1.7 & 26.7 \\
\hline & 13.748 & 1 & 1.7 & 1.7 & 28.3 \\
\hline & 13.801 & 1 & 1.7 & 1.7 & 30.0 \\
\hline & 13.843 & 1 & 1.7 & 1.7 & 31.7 \\
\hline & 13.970 & 1 & 1.7 & 1.7 & 33.3 \\
\hline & 14.217 & 1 & 1.7 & 1.7 & 35.0 \\
\hline & 14.274 & 1 & 1.7 & 1.7 & 36.7 \\
\hline & 14.295 & 1 & 1.7 & 1.7 & 38.3 \\
\hline & 14.330 & 1 & 1.7 & 1.7 & 40.0 \\
\hline & 14.334 & 1 & 1.7 & 1.7 & 41.7 \\
\hline & 14.447 & 1 & 1.7 & 1.7 & 43.3 \\
\hline & 14.500 & 1 & 1.7 & 1.7 & 45.0 \\
\hline & 14.542 & 1 & 1.7 & 1.7 & 46.7 \\
\hline & 14.751 & 1 & 1.7 & 1.7 & 48.3 \\
\hline & 14.864 & 1 & 1.7 & 1.7 & 50.0 \\
\hline & 14.881 & 1 & 1.7 & 1.7 & 51.7 \\
\hline & 14.902 & 1 & 1.7 & 1.7 & 53.3 \\
\hline & 14.966 & 1 & 1.7 & 1.7 & 55.0 \\
\hline & 15.054 & 1 & 1.7 & 1.7 & 56.7 \\
\hline & 15.072 & 1 & 1.7 & 1.7 & 58.3 \\
\hline & 15.142 & 1 & 1.7 & 1.7 & 60.0 \\
\hline & 15.160 & 1 & 1.7 & 1.7 & 61.7 \\
\hline & 15.174 & 1 & 1.7 & 1.7 & 63.3 \\
\hline & 15.386 & 1 & 1.7 & 1.7 & 65.0 \\
\hline & 15.488 & 1 & 1.7 & 1.7 & 66.7 \\
\hline & 15.506 & 1 & 1.7 & 1.7 & 68.3 \\
\hline & 15.626 & 1 & 1.7 & 1.7 & 70.0 \\
\hline & 15.644 & 1 & 1.7 & 1.7 & 71.7 \\
\hline & 15.665 & 1 & 1.7 & 1.7 & 73.3 \\
\hline & 16.029 & 1 & 1.7 & 1.7 & 75.0 \\
\hline & 16.131 & 1 & 1.7 & 1.7 & 76.7 \\
\hline & 16.195 & 1 & 1.7 & 1.7 & 78.3 \\
\hline & 16.308 & 1 & 1.7 & 1.7 & 80.0 \\
\hline & 16.428 & 1 & 1.7 & 1.7 & 81.7 \\
\hline & 16.491 & 1 & 1.7 & 1.7 & 83.3 \\
\hline & 16.519 & 1 & 1.7 & 1.7 & 85.0 \\
\hline & 16.745 & 2 & 3.3 & 3.3 & 88.3 \\
\hline & 16.819 & 1 & 1.7 & 1.7 & 90.0 \\
\hline & 16.943 & 1 & 1.7 & 1.7 & 91.7 \\
\hline & 17.105 & 1 & 1.7 & 1.7 & 93.3 \\
\hline & 17.162 & 1 & 1.7 & 1.7 & 95.0 \\
\hline & 17.621 & 1 & 1.7 & 1.7 & 96.7 \\
\hline & 17.826 & 1 & 1.7 & 1.7 & 98.3 \\
\hline & 17.974 & 1 & 1.7 & 1.7 & 100.0 \\
\hline & Total & 60 & 100.0 & 100.0 & \\
\hline
\end{tabular}




\begin{tabular}{|c|c|c|c|c|c|}
\hline \multicolumn{6}{|c|}{ Meanspeed Mean speed } \\
\hline & & Frequency & Percent & Valid Percent & $\begin{array}{c}\text { Cumulative } \\
\text { Percent }\end{array}$ \\
\hline \multirow[t]{47}{*}{ Valid } & .189 & 1 & 1.7 & 1.7 & 1.7 \\
\hline & .196 & 1 & 1.7 & 1.7 & 3.3 \\
\hline & .200 & 1 & 1.7 & 1.7 & 5.0 \\
\hline & .202 & 1 & 1.7 & 1.7 & 6.7 \\
\hline & .204 & 1 & 1.7 & 1.7 & 8.3 \\
\hline & .207 & 1 & 1.7 & 1.7 & 10.0 \\
\hline & .210 & 1 & 1.7 & 1.7 & 11.7 \\
\hline & .213 & 2 & 3.3 & 3.3 & 15.0 \\
\hline & .216 & 2 & 3.3 & 3.3 & 18.3 \\
\hline & .218 & 2 & 3.3 & 3.3 & 21.7 \\
\hline & .223 & 1 & 1.7 & 1.7 & 23.3 \\
\hline & .226 & 1 & 1.7 & 1.7 & 25.0 \\
\hline & .228 & 1 & 1.7 & 1.7 & 26.7 \\
\hline & .229 & 1 & 1.7 & 1.7 & 28.3 \\
\hline & .230 & 1 & 1.7 & 1.7 & 30.0 \\
\hline & .231 & 1 & 1.7 & 1.7 & 31.7 \\
\hline & .233 & 1 & 1.7 & 1.7 & 33.3 \\
\hline & .237 & 1 & 1.7 & 1.7 & 35.0 \\
\hline & .238 & 2 & 3.3 & 3.3 & 38.3 \\
\hline & .239 & 2 & 3.3 & 3.3 & 41.7 \\
\hline & .241 & 1 & 1.7 & 1.7 & 43.3 \\
\hline & .242 & 2 & 3.3 & 3.3 & 46.7 \\
\hline & .246 & 1 & 1.7 & 1.7 & 48.3 \\
\hline & .248 & 3 & 5.0 & 5.0 & 53.3 \\
\hline & .249 & 1 & 1.7 & 1.7 & 55.0 \\
\hline & .251 & 2 & 3.3 & 3.3 & 58.3 \\
\hline & .252 & 1 & 1.7 & 1.7 & 60.0 \\
\hline & .253 & 2 & 3.3 & 3.3 & 63.3 \\
\hline & .256 & 1 & 1.7 & 1.7 & 65.0 \\
\hline & .258 & 2 & 3.3 & 3.3 & 68.3 \\
\hline & .260 & 1 & 1.7 & 1.7 & 70.0 \\
\hline & .261 & 2 & 3.3 & 3.3 & 73.3 \\
\hline & .267 & 1 & 1.7 & 1.7 & 75.0 \\
\hline & .269 & 1 & 1.7 & 1.7 & 76.7 \\
\hline & .270 & 1 & 1.7 & 1.7 & 78.3 \\
\hline & .272 & 1 & 1.7 & 1.7 & 80.0 \\
\hline & .274 & 1 & 1.7 & 1.7 & 81.7 \\
\hline & .275 & 2 & 3.3 & 3.3 & 85.0 \\
\hline & .279 & 2 & 3.3 & 3.3 & 88.3 \\
\hline & .280 & 1 & 1.7 & 1.7 & 90.0 \\
\hline & .282 & 1 & 1.7 & 1.7 & 91.7 \\
\hline & .285 & 1 & 1.7 & 1.7 & 93.3 \\
\hline & .286 & 1 & 1.7 & 1.7 & 95.0 \\
\hline & .294 & 1 & 1.7 & 1.7 & 96.7 \\
\hline & .297 & 1 & 1.7 & 1.7 & 98.3 \\
\hline & .300 & 1 & 1.7 & 1.7 & 100.0 \\
\hline & Total & 60 & 100.0 & 100.0 & \\
\hline
\end{tabular}




\section{Maxspeed Max speed}

\begin{tabular}{|c|c|c|c|c|c|}
\hline & & Frequency & Percent & Valid Percent & $\begin{array}{c}\text { Cumulative } \\
\text { Percent }\end{array}$ \\
\hline \multirow[t]{40}{*}{ Valid } & .270 & 1 & 1.7 & 1.7 & 1.7 \\
\hline & .339 & 1 & 1.7 & 1.7 & 3.3 \\
\hline & .359 & 2 & 3.3 & 3.3 & 6.7 \\
\hline & .366 & 1 & 1.7 & 1.7 & 8.3 \\
\hline & .367 & 1 & 1.7 & 1.7 & 10.0 \\
\hline & .369 & 1 & 1.7 & 1.7 & 11.7 \\
\hline & .372 & 2 & 3.3 & 3.3 & 15.0 \\
\hline & .373 & 3 & 5.0 & 5.0 & 20.0 \\
\hline & .380 & 1 & 1.7 & 1.7 & 21.7 \\
\hline & .385 & 1 & 1.7 & 1.7 & 23.3 \\
\hline & .386 & 1 & 1.7 & 1.7 & 25.0 \\
\hline & .387 & 1 & 1.7 & 1.7 & 26.7 \\
\hline & .388 & 1 & 1.7 & 1.7 & 28.3 \\
\hline & .389 & 1 & 1.7 & 1.7 & 30.0 \\
\hline & .394 & 3 & 5.0 & 5.0 & 35.0 \\
\hline & .395 & 2 & 3.3 & 3.3 & 38.3 \\
\hline & .399 & 1 & 1.7 & 1.7 & 40.0 \\
\hline & .400 & 1 & 1.7 & 1.7 & 41.7 \\
\hline & .401 & 3 & 5.0 & 5.0 & 46.7 \\
\hline & .402 & 1 & 1.7 & 1.7 & 48.3 \\
\hline & .407 & 3 & 5.0 & 5.0 & 53.3 \\
\hline & .408 & 2 & 3.3 & 3.3 & 56.7 \\
\hline & .409 & 1 & 1.7 & 1.7 & 58.3 \\
\hline & .414 & 1 & 1.7 & 1.7 & 60.0 \\
\hline & .415 & 2 & 3.3 & 3.3 & 63.3 \\
\hline & .418 & 1 & 1.7 & 1.7 & 65.0 \\
\hline & .420 & 1 & 1.7 & 1.7 & 66.7 \\
\hline & .421 & 3 & 5.0 & 5.0 & 71.7 \\
\hline & .427 & 1 & 1.7 & 1.7 & 73.3 \\
\hline & .428 & 4 & 6.7 & 6.7 & 80.0 \\
\hline & .429 & 1 & 1.7 & 1.7 & 81.7 \\
\hline & .434 & 1 & 1.7 & 1.7 & 83.3 \\
\hline & .435 & 3 & 5.0 & 5.0 & 88.3 \\
\hline & .441 & 1 & 1.7 & 1.7 & 90.0 \\
\hline & .442 & 2 & 3.3 & 3.3 & 93.3 \\
\hline & .455 & 1 & 1.7 & 1.7 & 95.0 \\
\hline & .456 & 1 & 1.7 & 1.7 & 96.7 \\
\hline & .463 & 1 & 1.7 & 1.7 & 98.3 \\
\hline & .470 & 1 & 1.7 & 1.7 & 100.0 \\
\hline & Total & 60 & 100.0 & 100.0 & \\
\hline
\end{tabular}


ZONE1entries ZONE 1 : entries

\begin{tabular}{|c|c|c|c|c|c|}
\hline & & Frequency & Percent & Valid Percent & $\begin{array}{c}\text { Cumulative } \\
\text { Percent }\end{array}$ \\
\hline \multirow[t]{9}{*}{ Valid } & 2 & 1 & 1.7 & 1.7 & 1.7 \\
\hline & 3 & 9 & 15.0 & 15.0 & 16.7 \\
\hline & 4 & 11 & 18.3 & 18.3 & 35.0 \\
\hline & 5 & 16 & 26.7 & 26.7 & 61.7 \\
\hline & 6 & 9 & 15.0 & 15.0 & 76.7 \\
\hline & 7 & 8 & 13.3 & 13.3 & 90.0 \\
\hline & 8 & 5 & 8.3 & 8.3 & 98.3 \\
\hline & 10 & 1 & 1.7 & 1.7 & 100.0 \\
\hline & Total & 60 & 100.0 & 100.0 & \\
\hline
\end{tabular}




\begin{tabular}{|c|c|c|c|c|c|}
\hline \multicolumn{6}{|c|}{ ZONE1time ZONE 1 : time } \\
\hline & & Frequency & Percent & Valid Percent & $\begin{array}{c}\text { Cumulative } \\
\text { Percent }\end{array}$ \\
\hline \multirow[t]{54}{*}{ Valid } & 4.3 & 1 & 1.7 & 1.7 & 1.7 \\
\hline & 7.8 & 1 & 1.7 & 1.7 & 3.3 \\
\hline & 8.5 & 1 & 1.7 & 1.7 & 5.0 \\
\hline & 8.6 & 1 & 1.7 & 1.7 & 6.7 \\
\hline & 9.4 & 1 & 1.7 & 1.7 & 8.3 \\
\hline & 9.5 & 1 & 1.7 & 1.7 & 10.0 \\
\hline & 10.5 & 2 & 3.3 & 3.3 & 13.3 \\
\hline & 10.6 & 1 & 1.7 & 1.7 & 15.0 \\
\hline & 10.9 & 2 & 3.3 & 3.3 & 18.3 \\
\hline & 11.0 & 1 & 1.7 & 1.7 & 20.0 \\
\hline & 11.3 & 1 & 1.7 & 1.7 & 21.7 \\
\hline & 12.4 & 2 & 3.3 & 3.3 & 25.0 \\
\hline & 12.7 & 1 & 1.7 & 1.7 & 26.7 \\
\hline & 12.8 & 1 & 1.7 & 1.7 & 28.3 \\
\hline & 13.4 & 1 & 1.7 & 1.7 & 30.0 \\
\hline & 13.9 & 1 & 1.7 & 1.7 & 31.7 \\
\hline & 14.0 & 1 & 1.7 & 1.7 & 33.3 \\
\hline & 14.1 & 1 & 1.7 & 1.7 & 35.0 \\
\hline & 14.3 & 2 & 3.3 & 3.3 & 38.3 \\
\hline & 14.5 & 1 & 1.7 & 1.7 & 40.0 \\
\hline & 15.0 & 1 & 1.7 & 1.7 & 41.7 \\
\hline & 15.5 & 1 & 1.7 & 1.7 & 43.3 \\
\hline & 15.6 & 1 & 1.7 & 1.7 & 45.0 \\
\hline & 15.8 & 1 & 1.7 & 1.7 & 46.7 \\
\hline & 16.0 & 1 & 1.7 & 1.7 & 48.3 \\
\hline & 16.9 & 1 & 1.7 & 1.7 & 50.0 \\
\hline & 17.0 & 2 & 3.3 & 3.3 & 53.3 \\
\hline & 17.2 & 1 & 1.7 & 1.7 & 55.0 \\
\hline & 17.3 & 1 & 1.7 & 1.7 & 56.7 \\
\hline & 17.7 & 2 & 3.3 & 3.3 & 60.0 \\
\hline & 17.8 & 1 & 1.7 & 1.7 & 61.7 \\
\hline & 18.1 & 1 & 1.7 & 1.7 & 63.3 \\
\hline & 18.8 & 1 & 1.7 & 1.7 & 65.0 \\
\hline & 19.0 & 1 & 1.7 & 1.7 & 66.7 \\
\hline & 19.3 & 1 & 1.7 & 1.7 & 68.3 \\
\hline & 19.8 & 1 & 1.7 & 1.7 & 70.0 \\
\hline & 20.6 & 1 & 1.7 & 1.7 & 71.7 \\
\hline & 20.7 & 1 & 1.7 & 1.7 & 73.3 \\
\hline & 20.8 & 1 & 1.7 & 1.7 & 75.0 \\
\hline & 21.2 & 2 & 3.3 & 3.3 & 78.3 \\
\hline & 21.4 & 1 & 1.7 & 1.7 & 80.0 \\
\hline & 21.6 & 1 & 1.7 & 1.7 & 81.7 \\
\hline & 21.8 & 1 & 1.7 & 1.7 & 83.3 \\
\hline & 21.9 & 1 & 1.7 & 1.7 & 85.0 \\
\hline & 22.3 & 1 & 1.7 & 1.7 & 86.7 \\
\hline & 22.6 & 1 & 1.7 & 1.7 & 88.3 \\
\hline & 22.8 & 1 & 1.7 & 1.7 & 90.0 \\
\hline & 23.3 & 1 & 1.7 & 1.7 & 91.7 \\
\hline & 25.6 & 1 & 1.7 & 1.7 & 93.3 \\
\hline & 27.8 & 1 & 1.7 & 1.7 & 95.0 \\
\hline & 29.1 & 1 & 1.7 & 1.7 & 96.7 \\
\hline & 32.8 & 1 & 1.7 & 1.7 & 98.3 \\
\hline & 35.0 & 1 & 1.7 & 1.7 & 100.0 \\
\hline & Total & 60 & 100.0 & 100.0 & \\
\hline
\end{tabular}




\begin{tabular}{|c|c|c|c|c|c|}
\hline \multicolumn{6}{|c|}{ ZONE1distance ZONE 1 : distance } \\
\hline & & Frequency & Percent & Valid Percent & $\begin{array}{c}\text { Cumulative } \\
\text { Percent }\end{array}$ \\
\hline \multirow[t]{59}{*}{ Valid } & .999 & 1 & 1.7 & 1.7 & 1.7 \\
\hline & 2.065 & 1 & 1.7 & 1.7 & 3.3 \\
\hline & 2.263 & 1 & 1.7 & 1.7 & 5.0 \\
\hline & 2.401 & 1 & 1.7 & 1.7 & 6.7 \\
\hline & 2.450 & 1 & 1.7 & 1.7 & 8.3 \\
\hline & 2.475 & 1 & 1.7 & 1.7 & 10.0 \\
\hline & 2.683 & 1 & 1.7 & 1.7 & 11.7 \\
\hline & 2.690 & 1 & 1.7 & 1.7 & 13.3 \\
\hline & 2.750 & 1 & 1.7 & 1.7 & 15.0 \\
\hline & 2.856 & 1 & 1.7 & 1.7 & 16.7 \\
\hline & 2.948 & 1 & 1.7 & 1.7 & 18.3 \\
\hline & 2.980 & 1 & 1.7 & 1.7 & 20.0 \\
\hline & 3.033 & 1 & 1.7 & 1.7 & 21.7 \\
\hline & 3.107 & 1 & 1.7 & 1.7 & 23.3 \\
\hline & 3.113 & 1 & 1.7 & 1.7 & 25.0 \\
\hline & 3.160 & 1 & 1.7 & 1.7 & 26.7 \\
\hline & 3.167 & 1 & 1.7 & 1.7 & 28.3 \\
\hline & 3.280 & 1 & 1.7 & 1.7 & 30.0 \\
\hline & 3.294 & 1 & 1.7 & 1.7 & 31.7 \\
\hline & 3.315 & 1 & 1.7 & 1.7 & 33.3 \\
\hline & 3.396 & 1 & 1.7 & 1.7 & 35.0 \\
\hline & 3.442 & 1 & 1.7 & 1.7 & 36.7 \\
\hline & 3.492 & 1 & 1.7 & 1.7 & 38.3 \\
\hline & 3.612 & 1 & 1.7 & 1.7 & 40.0 \\
\hline & 3.647 & 1 & 1.7 & 1.7 & 41.7 \\
\hline & 3.668 & 1 & 1.7 & 1.7 & 43.3 \\
\hline & 3.813 & 1 & 1.7 & 1.7 & 45.0 \\
\hline & 3.827 & 1 & 1.7 & 1.7 & 46.7 \\
\hline & 3.944 & 1 & 1.7 & 1.7 & 48.3 \\
\hline & 3.951 & 1 & 1.7 & 1.7 & 50.0 \\
\hline & 4.233 & 2 & 3.3 & 3.3 & 53.3 \\
\hline & 4.258 & 1 & 1.7 & 1.7 & 55.0 \\
\hline & 4.279 & 1 & 1.7 & 1.7 & 56.7 \\
\hline & 4.304 & 1 & 1.7 & 1.7 & 58.3 \\
\hline & 4.353 & 1 & 1.7 & 1.7 & 60.0 \\
\hline & 4.427 & 2 & 3.3 & 3.3 & 63.3 \\
\hline & 4.456 & 1 & 1.7 & 1.7 & 65.0 \\
\hline & 4.491 & 1 & 1.7 & 1.7 & 66.7 \\
\hline & 4.565 & 1 & 1.7 & 1.7 & 68.3 \\
\hline & 4.625 & 1 & 1.7 & 1.7 & 70.0 \\
\hline & 4.710 & 1 & 1.7 & 1.7 & 71.7 \\
\hline & 4.756 & 1 & 1.7 & 1.7 & 73.3 \\
\hline & 4.766 & 1 & 1.7 & 1.7 & 75.0 \\
\hline & 4.773 & 1 & 1.7 & 1.7 & 76.7 \\
\hline & 4.861 & 1 & 1.7 & 1.7 & 78.3 \\
\hline & 4.914 & 1 & 1.7 & 1.7 & 80.0 \\
\hline & 4.964 & 1 & 1.7 & 1.7 & 81.7 \\
\hline & 4.978 & 1 & 1.7 & 1.7 & 83.3 \\
\hline & 5.087 & 1 & 1.7 & 1.7 & 85.0 \\
\hline & 5.091 & 1 & 1.7 & 1.7 & 86.7 \\
\hline & 5.236 & 1 & 1.7 & 1.7 & 88.3 \\
\hline & 5.681 & 1 & 1.7 & 1.7 & 90.0 \\
\hline & 5.857 & 1 & 1.7 & 1.7 & 91.7 \\
\hline & 6.195 & 1 & 1.7 & 1.7 & 93.3 \\
\hline & 6.217 & 1 & 1.7 & 1.7 & 95.0 \\
\hline & 6.612 & 1 & 1.7 & 1.7 & 96.7 \\
\hline & 6.719 & 1 & 1.7 & 1.7 & 98.3 \\
\hline & 7.329 & 1 & 1.7 & 1.7 & 100.0 \\
\hline & Total & 60 & 100.0 & 100.0 & \\
\hline
\end{tabular}


ZONE1latencytofirstentry ZONE 1 : latency to first entry

\begin{tabular}{|c|c|c|c|c|c|}
\hline & & Frequency & Percent & Valid Percent & $\begin{array}{c}\text { Cumulative } \\
\text { Percent }\end{array}$ \\
\hline \multirow[t]{55}{*}{ Valid } & .0 & 3 & 5.0 & 5.0 & 5.0 \\
\hline & .4 & 1 & 1.7 & 1.7 & 6.7 \\
\hline & .7 & 1 & 1.7 & 1.7 & 8.3 \\
\hline & .9 & 1 & 1.7 & 1.7 & 10.0 \\
\hline & 1.1 & 2 & 3.3 & 3.3 & 13.3 \\
\hline & 1.2 & 1 & 1.7 & 1.7 & 15.0 \\
\hline & 1.4 & 3 & 5.0 & 5.0 & 20.0 \\
\hline & 1.6 & 1 & 1.7 & 1.7 & 21.7 \\
\hline & 1.7 & 1 & 1.7 & 1.7 & 23.3 \\
\hline & 1.8 & 1 & 1.7 & 1.7 & 25.0 \\
\hline & 2.0 & 1 & 1.7 & 1.7 & 26.7 \\
\hline & 2.1 & 1 & 1.7 & 1.7 & 28.3 \\
\hline & 2.2 & 1 & 1.7 & 1.7 & 30.0 \\
\hline & 2.3 & 1 & 1.7 & 1.7 & 31.7 \\
\hline & 2.4 & 1 & 1.7 & 1.7 & 33.3 \\
\hline & 2.5 & 1 & 1.7 & 1.7 & 35.0 \\
\hline & 2.6 & 1 & 1.7 & 1.7 & 36.7 \\
\hline & 2.8 & 1 & 1.7 & 1.7 & 38.3 \\
\hline & 3.0 & 1 & 1.7 & 1.7 & 40.0 \\
\hline & 3.2 & 1 & 1.7 & 1.7 & 41.7 \\
\hline & 3.9 & 1 & 1.7 & 1.7 & 43.3 \\
\hline & 4.6 & 1 & 1.7 & 1.7 & 45.0 \\
\hline & 5.1 & 1 & 1.7 & 1.7 & 46.7 \\
\hline & 5.3 & 1 & 1.7 & 1.7 & 48.3 \\
\hline & 5.6 & 1 & 1.7 & 1.7 & 50.0 \\
\hline & 6.0 & 1 & 1.7 & 1.7 & 51.7 \\
\hline & 6.2 & 1 & 1.7 & 1.7 & 53.3 \\
\hline & 6.3 & 1 & 1.7 & 1.7 & 55.0 \\
\hline & 6.5 & 1 & 1.7 & 1.7 & 56.7 \\
\hline & 6.7 & 1 & 1.7 & 1.7 & 58.3 \\
\hline & 6.9 & 1 & 1.7 & 1.7 & 60.0 \\
\hline & 7.5 & 1 & 1.7 & 1.7 & 61.7 \\
\hline & 7.7 & 1 & 1.7 & 1.7 & 63.3 \\
\hline & 8.2 & 1 & 1.7 & 1.7 & 65.0 \\
\hline & 8.6 & 1 & 1.7 & 1.7 & 66.7 \\
\hline & 9.4 & 1 & 1.7 & 1.7 & 68.3 \\
\hline & 9.8 & 1 & 1.7 & 1.7 & 70.0 \\
\hline & 10.0 & 1 & 1.7 & 1.7 & 71.7 \\
\hline & 10.1 & 1 & 1.7 & 1.7 & 73.3 \\
\hline & 10.3 & 1 & 1.7 & 1.7 & 75.0 \\
\hline & 10.4 & 1 & 1.7 & 1.7 & 76.7 \\
\hline & 10.6 & 1 & 1.7 & 1.7 & 78.3 \\
\hline & 11.3 & 2 & 3.3 & 3.3 & 81.7 \\
\hline & 13.5 & 1 & 1.7 & 1.7 & 83.3 \\
\hline & 14.2 & 1 & 1.7 & 1.7 & 85.0 \\
\hline & 14.8 & 1 & 1.7 & 1.7 & 86.7 \\
\hline & 15.7 & 1 & 1.7 & 1.7 & 88.3 \\
\hline & 16.0 & 1 & 1.7 & 1.7 & 90.0 \\
\hline & 16.7 & 1 & 1.7 & 1.7 & 91.7 \\
\hline & 18.4 & 1 & 1.7 & 1.7 & 93.3 \\
\hline & 18.5 & 1 & 1.7 & 1.7 & 95.0 \\
\hline & 18.9 & 1 & 1.7 & 1.7 & 96.7 \\
\hline & 28.4 & 1 & 1.7 & 1.7 & 98.3 \\
\hline & 28.8 & 1 & 1.7 & 1.7 & 100.0 \\
\hline & Total & 60 & 100.0 & 100.0 & \\
\hline
\end{tabular}




\section{ZONE1maxspeed ZONE $1:$ max speed}

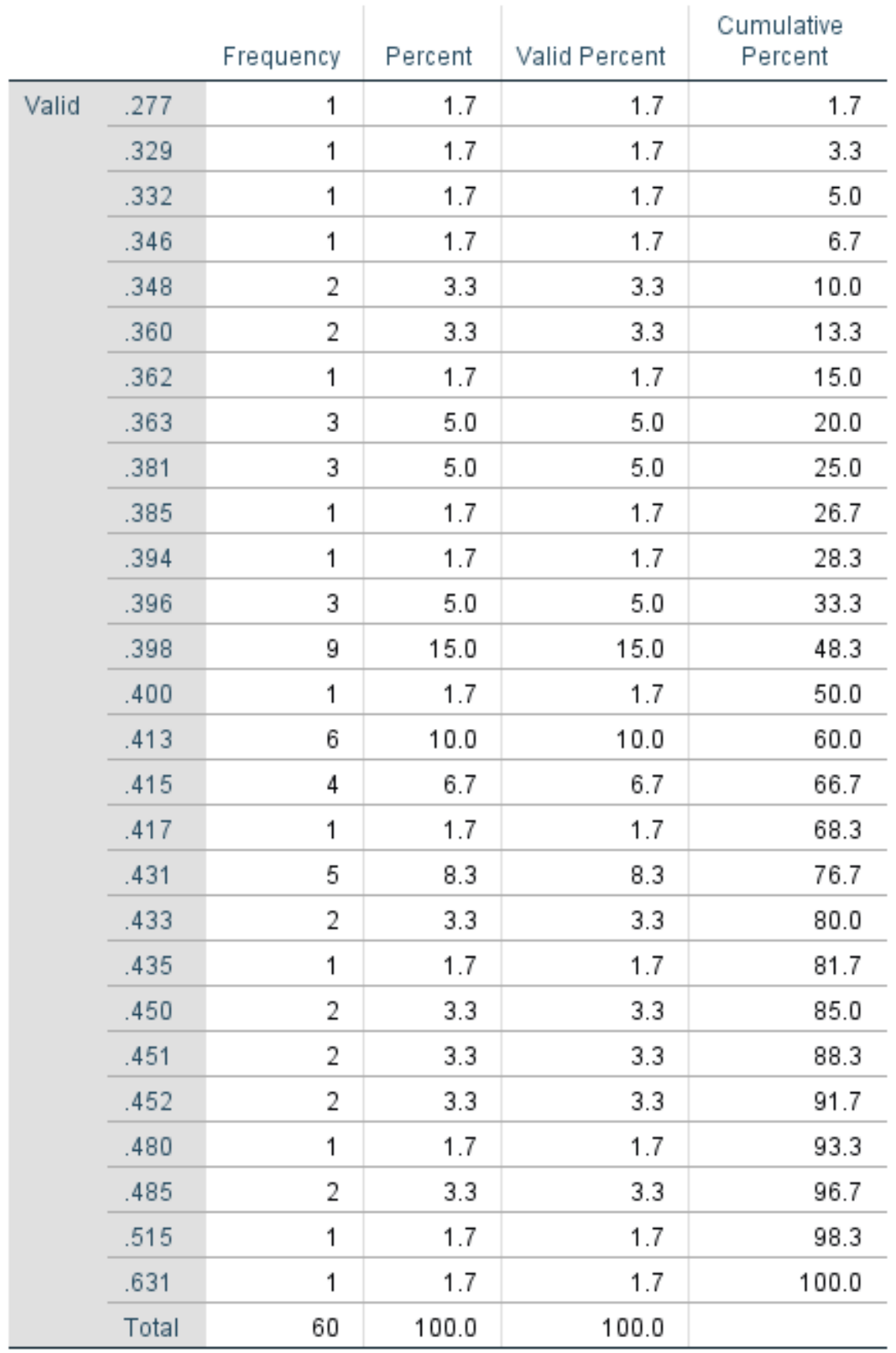



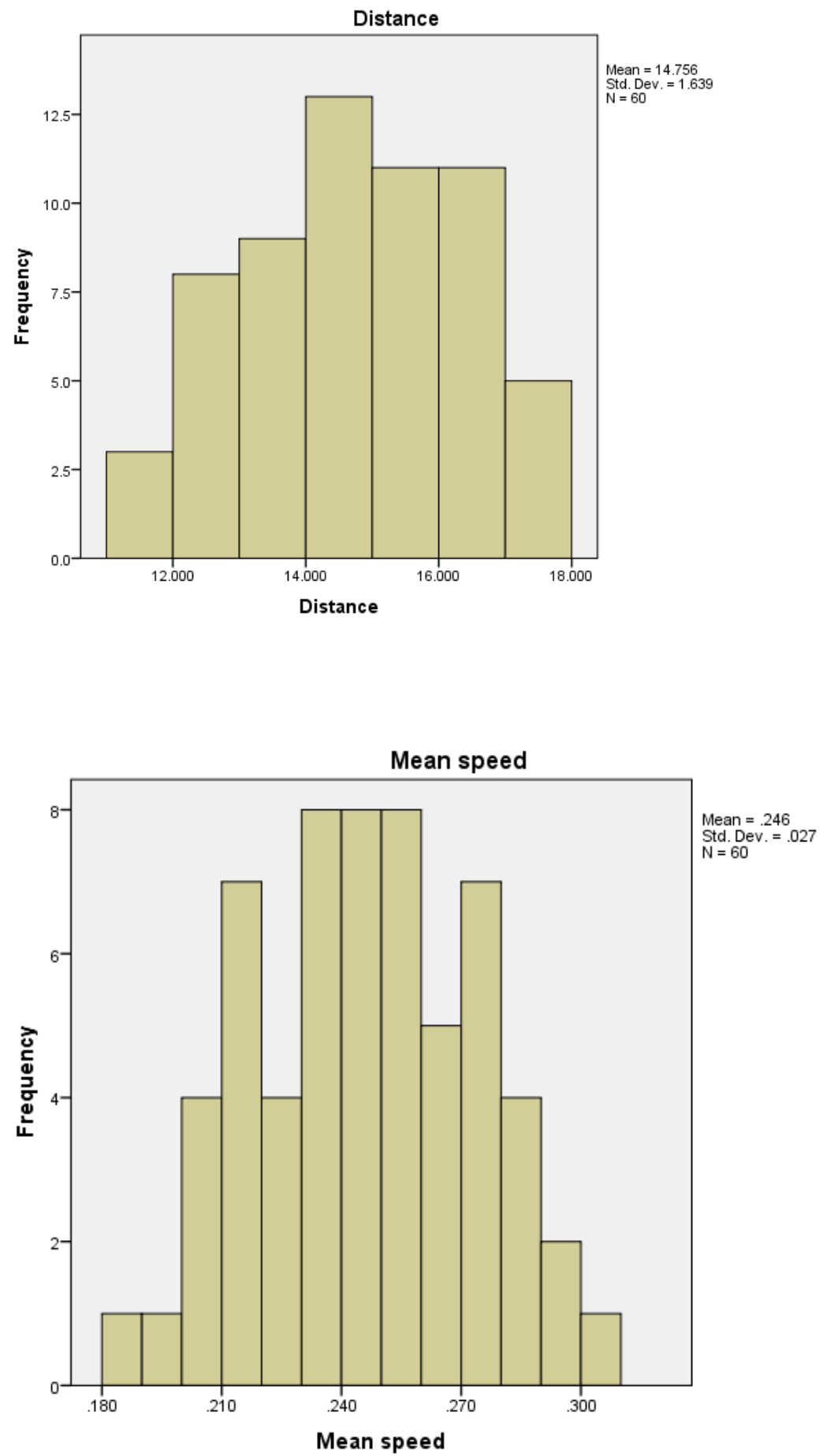

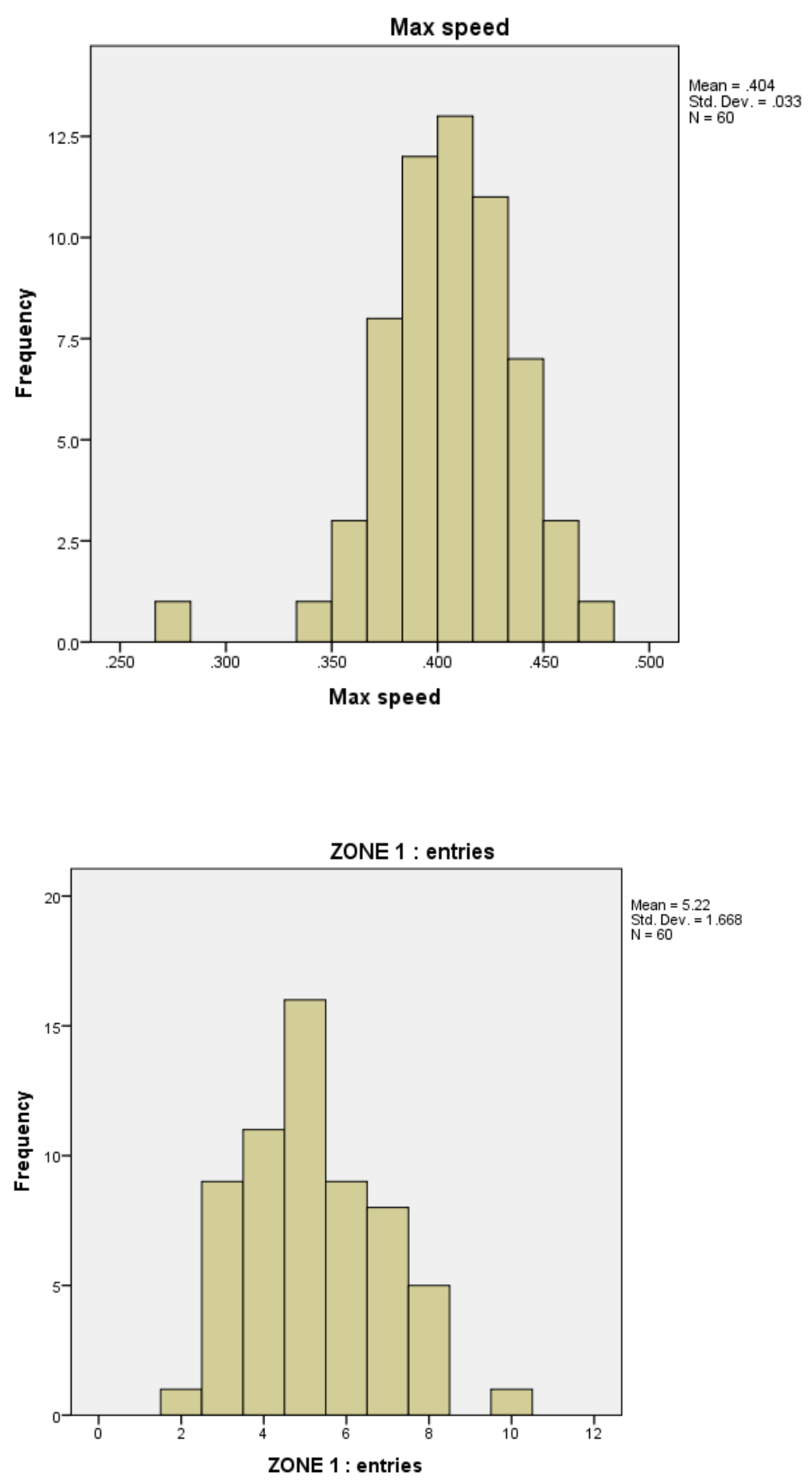

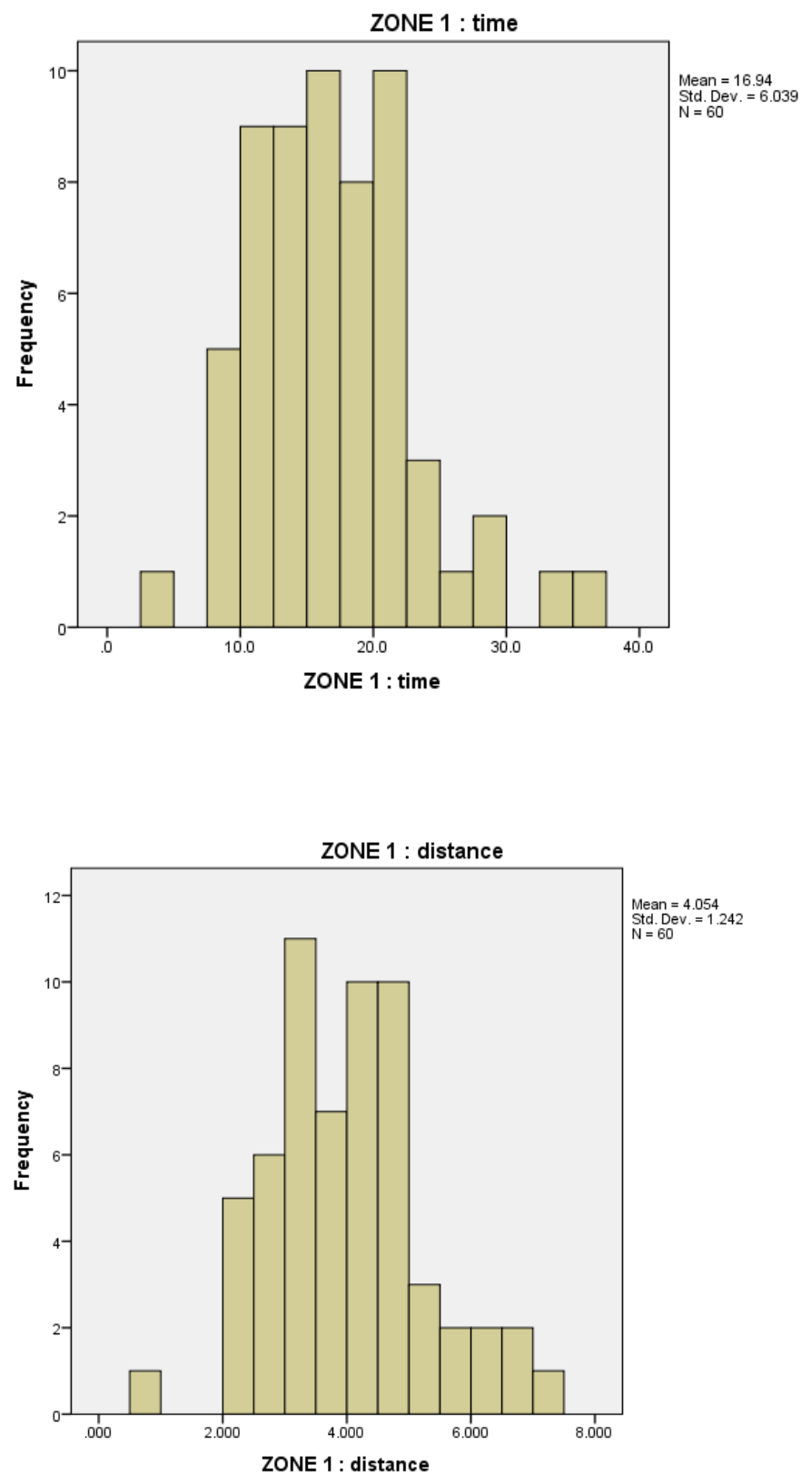

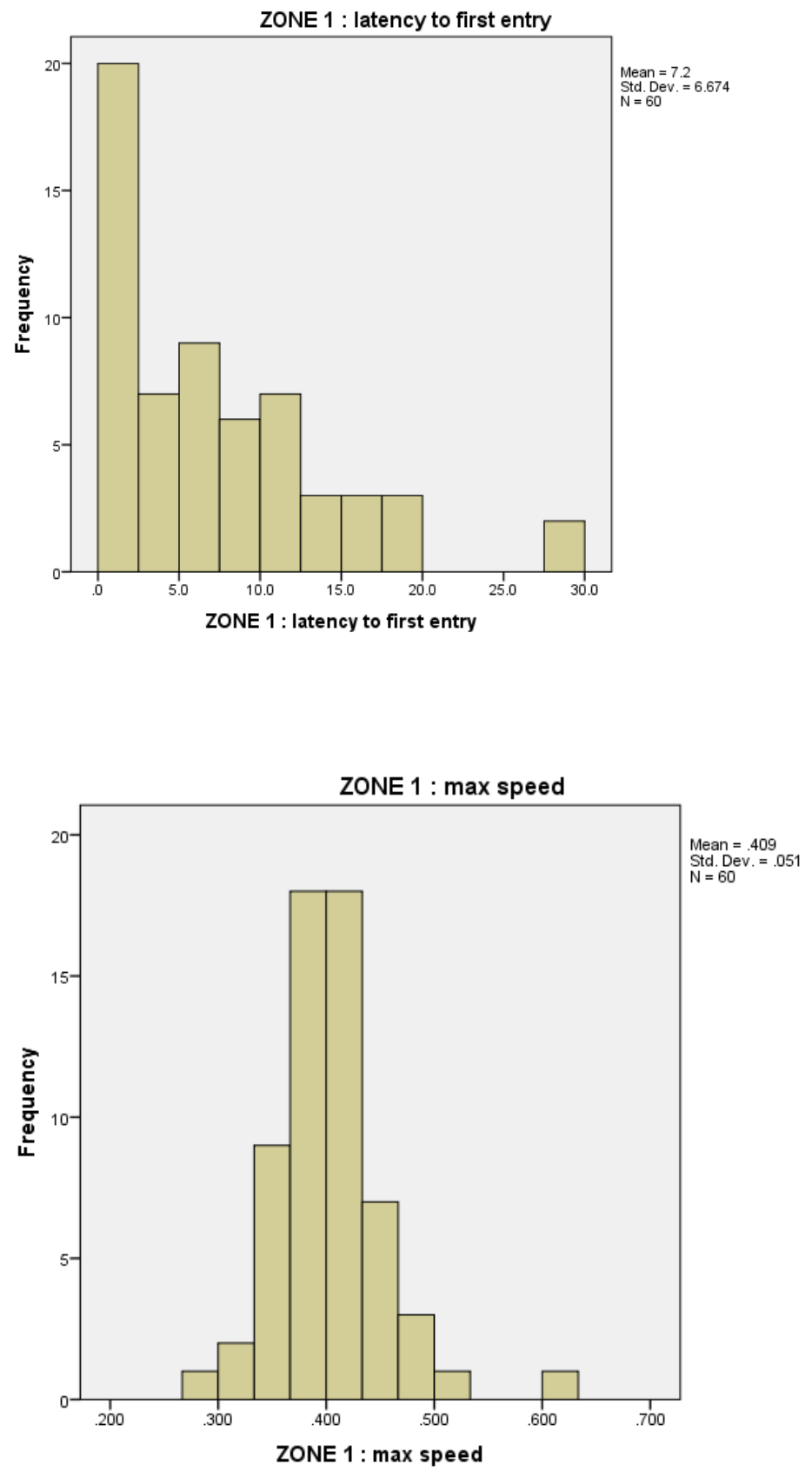


\section{By Group}

\begin{tabular}{|c|c|c|c|c|c|c|c|c|c|}
\hline \multicolumn{10}{|c|}{ Statistics $^{a}$} \\
\hline & & Distance & $\begin{array}{l}\text { Meanspeed } \\
\text { Mean speed }\end{array}$ & $\begin{array}{l}\text { Maxspeed } \\
\text { Max speed }\end{array}$ & $\begin{array}{l}\text { ZONE1 entrie } \\
\text { s ZONE 1: } \\
\text { entries }\end{array}$ & $\begin{array}{l}\text { ZONE1 time } \\
\text { ZONE } 1 \text { : time }\end{array}$ & $\begin{array}{l}\text { ZONE1 distan } \\
\text { ce ZONE 1: } \\
\text { distance }\end{array}$ & $\begin{array}{l}\text { ZONE1 latenc } \\
\text { ytofirstentry } \\
\text { ZONE 1: } \\
\text { latency to first } \\
\text { entry }\end{array}$ & $\begin{array}{l}\text { ZONE1 maxsp } \\
\text { eed ZONE } 1 \text { : } \\
\text { max speed }\end{array}$ \\
\hline \multirow[t]{2}{*}{ N } & Valid & 10 & 10 & 10 & 10 & 10 & 10 & 10 & 10 \\
\hline & Missing & 0 & 0 & 0 & 0 & 0 & 0 & 0 & 0 \\
\hline & & 14.55410 & .24260 & .41310 & 5.00 & 14.460 & 3.51650 & 9.760 & .41340 \\
\hline \multicolumn{2}{|c|}{ Std. Error of Mean } & .477502 & .007902 & .008703 & .745 & 1.8507 & .413659 & 2.6371 & .011006 \\
\hline \multicolumn{2}{|c|}{ Median } & 14.41700 & .24050 & .40850 & 4.50 & 14.100 & 3.10000 & 7.950 & .40550 \\
\hline \multicolumn{2}{|c|}{ Mode } & $12.092^{\mathrm{b}}$ & .239 & $.372^{\mathrm{b}}$ & $3^{b}$ & $7.8^{\mathrm{b}}$ & $2.065^{\mathrm{b}}$ & $1.1^{\mathrm{b}}$ & .398 \\
\hline \multicolumn{2}{|c|}{ Std. Deviation } & 1.509994 & .024990 & .027522 & 2.357 & 5.8523 & 1.308105 & 8.3394 & .034805 \\
\hline \multicolumn{2}{|c|}{ Variance } & 2.280 & .001 & .001 & 5.556 & 34.249 & 1.711 & 69.545 & .001 \\
\hline \multicolumn{2}{|c|}{ Skewness } & -.213 & -.208 & .706 & .955 & 1.260 & 1.771 & 1.291 & .850 \\
\hline \multicolumn{2}{|c|}{ Std. Error of Skewness } & .687 & .687 & .687 & .687 & .687 & .687 & .687 & .687 \\
\hline \multicolumn{2}{|c|}{ Kurtosis } & -.198 & -.189 & 1.043 & 1.021 & 2.344 & 3.985 & 1.780 & .938 \\
\hline \multicolumn{2}{|c|}{ Std. Error of Kurtosis } & 1.334 & 1.334 & 1.334 & 1.334 & 1.334 & 1.334 & 1.334 & 1.334 \\
\hline \multicolumn{2}{|c|}{ Range } & 4.727 & .078 & .098 & 8 & 20.0 & 4.654 & 27.3 & .122 \\
\hline \multicolumn{2}{|c|}{ Minimum } & 12.092 & .202 & .372 & 2 & 7.8 & 2.065 & 1.1 & .363 \\
\hline \multicolumn{2}{|c|}{ Maximum } & 16.819 & .280 & .470 & 10 & 27.8 & 6.719 & 28.4 & .485 \\
\hline \multicolumn{2}{|c|}{ Sum } & 145.541 & 2.426 & 4.131 & 50 & 144.6 & 35.165 & 97.6 & 4.134 \\
\hline
\end{tabular}

a. Group $=1$ control-vehicle

b. Multiple modes exist. The smallest value is shown

\begin{tabular}{|c|c|c|c|c|c|c|c|c|c|}
\hline \multicolumn{10}{|c|}{ Statistics $^{\mathrm{a}}$} \\
\hline & & Distance & $\begin{array}{l}\text { Meanspeed } \\
\text { Mean speed }\end{array}$ & $\begin{array}{l}\text { Maxspeed } \\
\text { Max speed }\end{array}$ & $\begin{array}{l}\text { ZONE1 entrie } \\
\text { s ZONE 1: } \\
\text { entries }\end{array}$ & $\begin{array}{l}\text { ZONE1 time } \\
\text { ZONE } 1 \text { : time }\end{array}$ & $\begin{array}{l}\text { ZONE1 distan } \\
\text { ce ZONE 1: } \\
\text { distance }\end{array}$ & $\begin{array}{l}\text { ZONE1 latenc } \\
\text { ytofirstentry } \\
\text { ZONE 1: } \\
\text { latency to first } \\
\text { entry }\end{array}$ & $\begin{array}{l}\text { ZONE1 maxsp } \\
\text { eed ZONE 1: } \\
\text { max speed }\end{array}$ \\
\hline \multirow[t]{2}{*}{$\mathrm{N}$} & Valid & 10 & 10 & 10 & 10 & 10 & 10 & 10 & 10 \\
\hline & Missing & 0 & 0 & 0 & 0 & 0 & 0 & 0 & 0 \\
\hline \multicolumn{2}{|c|}{ Mean } & 14.87760 & .24790 & .41430 & 4.70 & 15.200 & 3.69440 & 10.670 & .40460 \\
\hline \multicolumn{2}{|c|}{ Std. Error of Mean } & .480676 & .007984 & .006838 & .423 & 1.5101 & .383844 & 2.6110 & .015248 \\
\hline \multicolumn{2}{|c|}{ Median } & 14.97650 & .24950 & .41250 & 4.50 & 15.750 & 3.55200 & 10.050 & .41300 \\
\hline \multicolumn{2}{|c|}{ Mode } & $12.936^{\mathrm{b}}$ & $.216^{\mathrm{b}}$ & .407 & 4 & $8.6^{\mathrm{b}}$ & $2.263^{\mathrm{b}}$ & $.0^{\mathrm{b}}$ & .413 \\
\hline \multicolumn{2}{|c|}{ Std. Deviation } & 1.520030 & .025247 & .021623 & 1.337 & 4.7754 & 1.213820 & 8.2569 & .048220 \\
\hline \multicolumn{2}{|c|}{ Variance } & 2.310 & .001 & .000 & 1.789 & 22.804 & 1.473 & 68.176 & .002 \\
\hline \multicolumn{2}{|c|}{ Skewness } & .611 & .615 & -.072 & .334 & -.080 & .124 & 1.096 & -.096 \\
\hline \multicolumn{2}{|c|}{ Std. Error of Skewness } & .687 & .687 & .687 & .687 & .687 & .687 & .687 & .687 \\
\hline \multicolumn{2}{|c|}{ Kurtosis } & .121 & .145 & -1.538 & -.852 & -1.627 & -1.985 & 1.917 & -.491 \\
\hline \multicolumn{2}{|c|}{ Std. Error of Kurtosis } & 1.334 & 1.334 & 1.334 & 1.334 & 1.334 & 1.334 & 1.334 & 1.334 \\
\hline \multicolumn{2}{|c|}{ Range } & 4.890 & .081 & .057 & 4 & 13.0 & 2.973 & 28.8 & .156 \\
\hline \multicolumn{2}{|c|}{ Minimum } & 12.936 & .216 & .385 & 3 & 8.6 & 2.263 & .0 & .329 \\
\hline \multicolumn{2}{|c|}{ Maximum } & 17.826 & .297 & .442 & 7 & 21.6 & 5.236 & 28.8 & .485 \\
\hline \multicolumn{2}{|c|}{ Sum } & 148.776 & 2.479 & 4.143 & 47 & 152.0 & 36.944 & 106.7 & 4.046 \\
\hline
\end{tabular}

a. Group $=2 \mathrm{~L}$-Th control drug

b. Multiple modes exist. The smallest value is shown 


\begin{tabular}{|c|c|c|c|c|c|c|c|c|c|}
\hline \multicolumn{10}{|c|}{ Statistics $^{a}$} \\
\hline & & Distance & $\begin{array}{l}\text { Meanspeed } \\
\text { Mean speed }\end{array}$ & $\begin{array}{l}\text { Maxspeed } \\
\text { Max speed }\end{array}$ & $\begin{array}{l}\text { ZONE1 entrie } \\
\text { s ZONE 1: } \\
\text { entries }\end{array}$ & $\begin{array}{l}\text { ZONE1 time } \\
\text { ZONE 1: time }\end{array}$ & $\begin{array}{l}\text { ZONE1 distan } \\
\text { ce ZONE 1: } \\
\text { distance }\end{array}$ & $\begin{array}{l}\text { ZONE1 latenc } \\
\text { ytofirstentry } \\
\text { ZONE 1: } \\
\text { latency to first } \\
\text { entry }\end{array}$ & $\begin{array}{l}\text { ZONE1 maxsp } \\
\text { eed ZONE } 1 \text { : } \\
\text { max speed }\end{array}$ \\
\hline \multirow[t]{2}{*}{$\mathrm{N}$} & Valid & 10 & 10 & 10 & 10 & 10 & 10 & 10 & 10 \\
\hline & Missing & 0 & 0 & 0 & 0 & 0 & 0 & 0 & 0 \\
\hline \multicolumn{2}{|c|}{ Mean } & 15.04290 & .25060 & .41240 & 5.30 & 19.370 & 4.53590 & 4.540 & .40400 \\
\hline \multicolumn{2}{|c|}{ Std. Error of Mean } & .419332 & .007030 & .011905 & .559 & 1.5300 & .363886 & 2.0355 & .012903 \\
\hline \multicolumn{2}{|c|}{ Median } & 15.05400 & .25050 & .41350 & 5.50 & 18.800 & 4.39000 & 1.400 & .39600 \\
\hline \multicolumn{2}{|c|}{ Mode } & $13.102^{b}$ & $.218^{\mathrm{b}}$ & $.369^{\mathrm{b}}$ & $3^{b}$ & $12.4^{\mathrm{b}}$ & $3.294^{\mathrm{b}}$ & 1.4 & .381 \\
\hline \multicolumn{2}{|c|}{ Std. Deviation } & 1.326043 & .022232 & .037648 & 1.767 & 4.8383 & 1.150709 & 6.4369 & .040803 \\
\hline \multicolumn{2}{|c|}{ Variance } & 1.758 & .000 & .001 & 3.122 & 23.409 & 1.324 & 41.434 & .002 \\
\hline \multicolumn{2}{|c|}{ Skewness } & .461 & .482 & .135 & .036 & .602 & 1.632 & 1.689 & .603 \\
\hline \multicolumn{2}{|c|}{ Std. Error of Skewness } & .687 & .687 & .687 & .687 & .687 & .687 & .687 & .687 \\
\hline \multicolumn{2}{|c|}{ Kurtosis } & .272 & .314 & -1.812 & -1.384 & .545 & 3.827 & 1.614 & -.188 \\
\hline \multicolumn{2}{|c|}{ Std. Error of Kurtosis } & 1.334 & 1.334 & 1.334 & 1.334 & 1.334 & 1.334 & 1.334 & 1.334 \\
\hline \multicolumn{2}{|c|}{ Range } & 4.519 & .076 & .094 & 5 & 16.7 & 4.035 & 18.4 & .132 \\
\hline \multicolumn{2}{|c|}{ Minimum } & 13.102 & .218 & .369 & 3 & 12.4 & 3.294 & .0 & .348 \\
\hline \multicolumn{2}{|c|}{ Maximum } & 17.621 & .294 & .463 & 8 & 29.1 & 7.329 & 18.4 & .480 \\
\hline \multicolumn{2}{|c|}{ Sum } & 150.429 & 2.506 & 4.124 & 53 & 193.7 & 45.359 & 45.4 & 4.040 \\
\hline
\end{tabular}

a. Group $=3$ control-naïve

b. Multiple modes exist. The smallest value is shown

\begin{tabular}{|c|c|c|c|c|c|c|c|c|c|}
\hline \multicolumn{10}{|c|}{ Statistics $^{a}$} \\
\hline & & Distance & $\begin{array}{l}\text { Meanspeed } \\
\text { Mean speed }\end{array}$ & $\begin{array}{l}\text { Maxspeed } \\
\text { Max speed }\end{array}$ & $\begin{array}{l}\text { ZONE1 entrie } \\
\text { s ZONE 1: } \\
\text { entries }\end{array}$ & $\begin{array}{l}\text { ZONE1 time } \\
\text { ZONE 1: time }\end{array}$ & $\begin{array}{l}\text { ZONE1 distan } \\
\text { ce ZONE 1: } \\
\text { distance }\end{array}$ & $\begin{array}{l}\text { ZONE1 latenc } \\
\text { ytofirstentry } \\
\text { ZONE 1: } \\
\text { latency to first } \\
\text { entry }\end{array}$ & $\begin{array}{l}\text { ZONE1 maxsp } \\
\text { eed ZONE } 1 \text { : } \\
\text { max speed }\end{array}$ \\
\hline \multirow[t]{2}{*}{$N$} & Valid & 10 & 10 & 10 & 10 & 10 & 10 & 10 & 10 \\
\hline & Missing & 0 & 0 & 0 & 0 & 0 & 0 & 0 & 0 \\
\hline & & 15.28050 & .25470 & .39990 & 5.30 & 17.090 & 4.16040 & 5.140 & .39360 \\
\hline \multicolumn{2}{|c|}{ Std. Error of Mean } & .479612 & .007940 & .010308 & .396 & 1.7413 & .299289 & 1.5988 & .009057 \\
\hline \multicolumn{2}{|c|}{ Median } & 15.54150 & .25900 & .40750 & 5.00 & 16.300 & 4.37450 & 3.500 & .39800 \\
\hline \multicolumn{2}{|c|}{ Mode } & 16.745 & .279 & $.401^{\mathrm{b}}$ & 5 & $10.5^{\mathrm{b}}$ & $2.683^{\mathrm{b}}$ & $1.6^{\mathrm{b}}$ & .398 \\
\hline \multicolumn{2}{|c|}{ Std. Deviation } & 1.516665 & .025109 & .032597 & 1.252 & 5.5065 & .946436 & 5.0557 & .028640 \\
\hline \multicolumn{2}{|c|}{ Variance } & 2.300 & .001 & .001 & 1.567 & 30.321 & .896 & 25.560 & .001 \\
\hline \multicolumn{2}{|c|}{ Skewness } & -.474 & -.470 & -.858 & -.280 & .214 & -.227 & 2.400 & -.291 \\
\hline \multicolumn{2}{|c|}{ Std. Error of Skewness } & .687 & .687 & .687 & .687 & .687 & .687 & .687 & .687 \\
\hline \multicolumn{2}{|c|}{ Kurtosis } & -1.262 & -1.286 & -.458 & -.066 & -1.508 & -.695 & 6.469 & -.923 \\
\hline \multicolumn{2}{|c|}{ Std. Error of Kurtosis } & 1.334 & 1.334 & 1.334 & 1.334 & 1.334 & 1.334 & 1.334 & 1.334 \\
\hline \multicolumn{2}{|c|}{ Range } & 4.191 & .069 & .096 & 4 & 15.1 & 2.998 & 16.9 & .083 \\
\hline \multicolumn{2}{|c|}{ Minimum } & 12.752 & .213 & .339 & 3 & 10.5 & 2.683 & 1.6 & .348 \\
\hline \multicolumn{2}{|c|}{ Maximum } & 16.943 & .282 & .435 & 7 & 25.6 & 5.681 & 18.5 & .431 \\
\hline \multicolumn{2}{|c|}{ Sum } & 152.805 & 2.547 & 3.999 & 53 & 170.9 & 41.604 & 51.4 & 3.936 \\
\hline
\end{tabular}

a. Group $=4$ PTSD-vehicle

b. Multiple modes exist. The smallest value is shown 


\begin{tabular}{|c|c|c|c|c|c|c|c|c|c|}
\hline \multicolumn{10}{|c|}{ Statistics $^{a}$} \\
\hline & & Distance & $\begin{array}{l}\text { Meanspeed } \\
\text { Mean speed }\end{array}$ & $\begin{array}{l}\text { Maxspeed } \\
\text { Max speed }\end{array}$ & $\begin{array}{l}\text { ZONE1 entrie } \\
\text { s ZONE } 1: \\
\text { entries }\end{array}$ & $\begin{array}{l}\text { ZONE1 time } \\
\text { ZONE } 1 \text { : time }\end{array}$ & $\begin{array}{l}\text { ZONE1 distan } \\
\text { ce ZONE 1: } \\
\text { distance }\end{array}$ & $\begin{array}{l}\text { ZONE1 latenc } \\
\text { ytofirstentry } \\
\text { ZONE 1: } \\
\text { latency to first } \\
\text { entry }\end{array}$ & $\begin{array}{l}\text { ZONE1 maxsp } \\
\text { eed ZONE } 1 \text { : } \\
\text { max speed }\end{array}$ \\
\hline \multirow[t]{2}{*}{$\mathrm{N}$} & Valid & 10 & 10 & 10 & 10 & 10 & 10 & 10 & 10 \\
\hline & Missing & 0 & 0 & 0 & 0 & 0 & 0 & 0 & 0 \\
\hline \multicolumn{2}{|c|}{ Mean } & 13.62540 & .22700 & .39510 & 5.10 & 19.180 & 4.23490 & 7.090 & .42180 \\
\hline \multicolumn{2}{|c|}{ Std. Error of Mean } & .572799 & .009581 & .006049 & .526 & 2.7523 & .424618 & 1.8641 & .015319 \\
\hline \multicolumn{2}{|c|}{ Median } & 13.19250 & .21950 & .39400 & 5.00 & 17.000 & 3.83750 & 4.500 & .42300 \\
\hline \multicolumn{2}{|c|}{ Mode } & $11.347^{b}$ & $.189^{b}$ & $.394^{b}$ & 5 & $10.5^{\mathrm{b}}$ & $2.750^{\mathrm{b}}$ & 11.3 & .451 \\
\hline \multicolumn{2}{|c|}{ Std. Deviation } & 1.811349 & .030299 & .019128 & 1.663 & 8.7035 & 1.342761 & 5.8949 & .048442 \\
\hline \multicolumn{2}{|c|}{ Variance } & 3.281 & .001 & .000 & 2.767 & 75.751 & 1.803 & 34.750 & .002 \\
\hline \multicolumn{2}{|c|}{ Skewness } & .627 & .621 & .680 & 1.072 & 1.000 & .814 & .555 & .063 \\
\hline \multicolumn{2}{|c|}{ Std. Error of Skewness } & .687 & .687 & .687 & .687 & .687 & .687 & .687 & .687 \\
\hline \multicolumn{2}{|c|}{ Kurtosis } & -.285 & -.323 & 1.295 & .374 & -.130 & -.588 & -1.334 & 1.415 \\
\hline \multicolumn{2}{|c|}{ Std. Error of Kurtosis } & 1.334 & 1.334 & 1.334 & 1.334 & 1.334 & 1.334 & 1.334 & 1.334 \\
\hline \multicolumn{2}{|c|}{ Range } & 5.758 & .096 & .068 & 5 & 24.5 & 3.862 & 16.7 & .183 \\
\hline \multicolumn{2}{|c|}{ Minimum } & 11.347 & .189 & .367 & 3 & 10.5 & 2.750 & .0 & .332 \\
\hline \multicolumn{2}{|c|}{ Maximum } & 17.105 & .285 & .435 & 8 & 35.0 & 6.612 & 16.7 & .515 \\
\hline \multicolumn{2}{|c|}{ Sum } & 136.254 & 2.270 & 3.951 & 51 & 191.8 & 42.349 & 70.9 & 4.218 \\
\hline
\end{tabular}

a. Group $=5$ PTSD-drug pre-treatment

b. Multiple modes exist. The smallest value is shown

\begin{tabular}{|c|c|c|c|c|c|c|c|c|c|}
\hline \multicolumn{10}{|c|}{ Statistics $^{\mathrm{a}}$} \\
\hline & & Distance & $\begin{array}{l}\text { Meanspeed } \\
\text { Mean speed }\end{array}$ & $\begin{array}{l}\text { Maxspeed } \\
\text { Max speed }\end{array}$ & $\begin{array}{l}\text { ZONE1 entrie } \\
\text { s ZONE 1: } \\
\text { entries }\end{array}$ & $\begin{array}{l}\text { ZONE1 time } \\
\text { ZONE 1: time }\end{array}$ & $\begin{array}{l}\text { ZONE1 distan } \\
\text { ce ZONE 1: } \\
\text { distance }\end{array}$ & $\begin{array}{l}\text { ZONE1 latenc } \\
\text { ytofirstentry } \\
\text { ZONE 1: } \\
\text { latency to first } \\
\text { entry }\end{array}$ & $\begin{array}{l}\text { ZONE1 maxsp } \\
\text { eed ZONE } 1 \text { : } \\
\text { max speed }\end{array}$ \\
\hline \multirow[t]{2}{*}{$\mathrm{N}$} & Valid & 10 & 10 & 10 & 10 & 10 & 10 & 10 & 10 \\
\hline & Missing & 0 & 0 & 0 & 0 & 0 & 0 & 0 & 0 \\
\hline \multicolumn{2}{|c|}{ Mean } & 15.15620 & .25270 & .39020 & 5.90 & 16.360 & 4.17970 & 6.000 & .41820 \\
\hline \multicolumn{2}{|c|}{ Std. Error of Mean } & .601780 & .010034 & .015654 & .504 & 1.7261 & .460614 & 1.3301 & .028047 \\
\hline \multicolumn{2}{|c|}{ Median } & 15.57650 & .25950 & .40400 & 5.50 & 17.350 & 4.33000 & 6.450 & .40750 \\
\hline \multicolumn{2}{|c|}{ Mode } & $11.972^{b}$ & $.200^{\mathrm{b}}$ & $.270^{\mathrm{b}}$ & 5 & $4.3^{\mathrm{b}}$ & $.999^{\mathrm{b}}$ & $.7^{\mathrm{b}}$ & $.398^{\mathrm{b}}$ \\
\hline \multicolumn{2}{|c|}{ Std. Deviation } & 1.902997 & .031732 & .049502 & 1.595 & 5.4584 & 1.456588 & 4.2061 & .088693 \\
\hline \multicolumn{2}{|c|}{ Variance } & 3.621 & .001 & .002 & 2.544 & 29.794 & 2.122 & 17.691 & .008 \\
\hline \multicolumn{2}{|c|}{ Skewness } & -.276 & -.260 & -1.764 & -.209 & -1.244 & -.898 & .327 & 1.328 \\
\hline \multicolumn{2}{|c|}{ Std. Error of Skewness } & .687 & .687 & .687 & .687 & .687 & .687 & .687 & .687 \\
\hline \multicolumn{2}{|c|}{ Kurtosis } & -.795 & -.807 & 3.675 & -.457 & 1.713 & 1.919 & -.687 & 4.334 \\
\hline \multicolumn{2}{|c|}{ Std. Error of Kurtosis } & 1.334 & 1.334 & 1.334 & 1.334 & 1.334 & 1.334 & 1.334 & 1.334 \\
\hline \multicolumn{2}{|c|}{ Range } & 6.002 & .100 & .172 & 5 & 18.5 & 5.218 & 12.8 & .354 \\
\hline \multicolumn{2}{|c|}{ Minimum } & 11.972 & .200 & .270 & 3 & 4.3 & .999 & .7 & .277 \\
\hline \multicolumn{2}{|c|}{ Maximum } & 17.974 & .300 & .442 & 8 & 22.8 & 6.217 & 13.5 & .631 \\
\hline \multicolumn{2}{|c|}{ Sum } & 151.562 & 2.527 & 3.902 & 59 & 163.6 & 41.797 & 60.0 & 4.182 \\
\hline
\end{tabular}

a. Group $=6$ PTSD-post-treatment

b. Multiple modes exist. The smallest value is shown 


\section{One-Way ANOVA}

\section{Distance}

\begin{tabular}{|c|c|c|c|}
\hline \multicolumn{4}{|c|}{ Descriptive Statistics } \\
\hline Dependent Variable: D & nce & & \\
\hline Group & Mean & Std. Deviation & $N$ \\
\hline 1 control-vehicle & 14.55410 & 1.509994 & 10 \\
\hline $2 \mathrm{~L}$-Th control drug & 14.87760 & 1.520030 & 10 \\
\hline 3 control-naïve & 15.04290 & 1.326043 & 10 \\
\hline 4 PTSD-vehicle & 15.28050 & 1.516665 & 10 \\
\hline $\begin{array}{l}5 \text { PTSD-drug pre- } \\
\text { treatment }\end{array}$ & 13.62540 & 1.811349 & 10 \\
\hline 6 PTSD-post-treatment & 15.15620 & 1.902997 & 10 \\
\hline Total & 14.75612 & 1.638925 & 60 \\
\hline
\end{tabular}

\section{Levene's Test of Equality of Error \\ Variances $^{\mathrm{a}}$}

Dependent Variable: Distance

\begin{tabular}{r|r|r|r}
$\mathrm{F}$ & $\mathrm{df1}$ & $\mathrm{df2}$ & \multicolumn{1}{c}{ Sig. } \\
\hline .644 & 5 & 54 & .667 \\
\hline
\end{tabular}

Tests the null hypothesis that the error variance of the dependent variable is equal across groups.

a. Design: Intercept + Group

\section{Tests of Between-Subjects Effects}

Dependent Variable: Distance

\begin{tabular}{|c|c|c|c|c|c|c|}
\hline Source & $\begin{array}{c}\text { Type III Sum } \\
\text { of Squares }\end{array}$ & df & Mean Square & $\mathrm{F}$ & Sig. & $\begin{array}{c}\text { Partial Eta } \\
\text { Squared }\end{array}$ \\
\hline Corrected Model & $18.514^{a}$ & 5 & 3.703 & 1.429 & .229 & .117 \\
\hline Intercept & 13064.579 & 1 & 13064.579 & 5040.470 & .000 & .989 \\
\hline Group & 18.514 & 5 & 3.703 & 1.429 & .229 & .117 \\
\hline Error & 139.965 & 54 & 2.592 & & & \\
\hline Total & 13223.057 & 60 & & & & \\
\hline Corrected Total & 158.478 & 59 & & & & \\
\hline
\end{tabular}

a. $\mathrm{R}$ Squared $=.117$ (Adjusted R Squared $=.035$ ) 


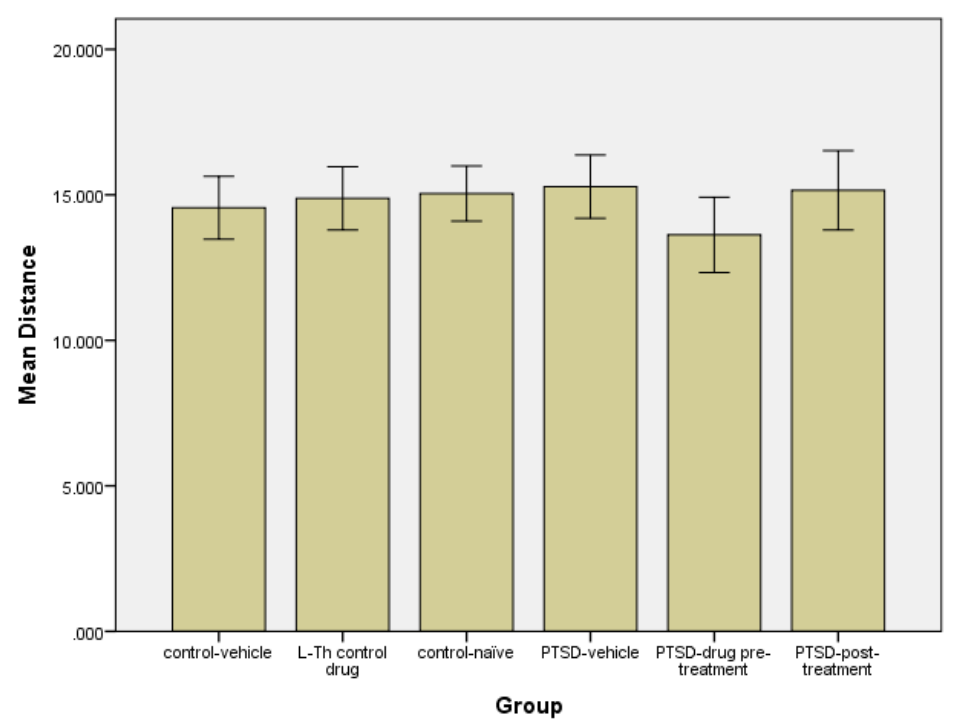

Error bars: $95 \% \mathrm{Cl}$

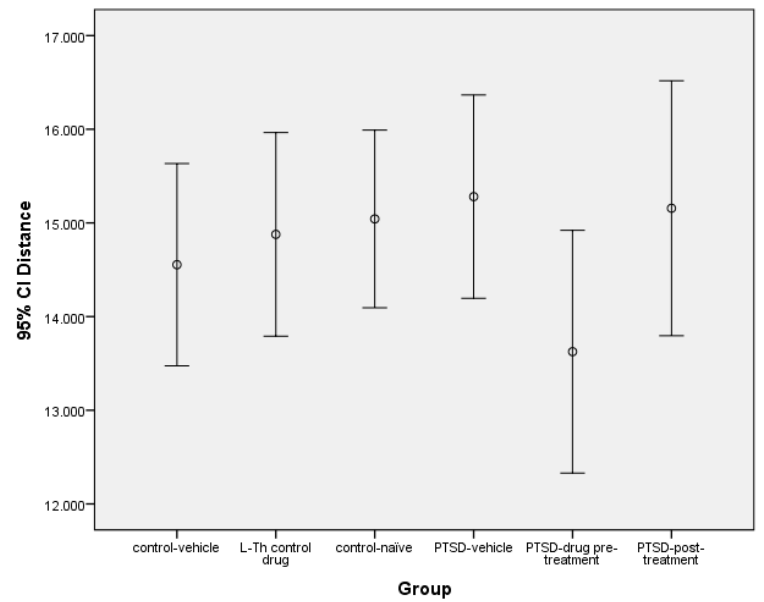

Summary: For the distance outcome, there was not a significant difference between the six groups: $F(5,54)=1.43, p=.229\left(\eta^{2}=.117\right)$. Though not significant the PTSD-vehicle group has the highest mean $(M=15.28)$ and PTSD-drug pre-treatment the lowest $(M=16.63)$. 


\section{Mean Speed}

\section{Descriptive Statistics}

Dependent Variable: Meanspeed Mean speed

\begin{tabular}{ll|r|r} 
Group & Mean & Std. Deviation & \multicolumn{1}{l}{$\mathrm{N}$} \\
\hline 1 control-vehicle & .24260 & .024990 & 10 \\
\hline 2 L-Th control drug & .24790 & .025247 & 10 \\
\hline 3 control-naïve & .25060 & .022232 & 10 \\
\hline 4 PTSD-vehicle & .25470 & .025109 & 10 \\
\hline $\begin{array}{l}\text { 5 PTSD-drug pre- } \\
\text { treatment }\end{array}$ & .22700 & .030299 & 10 \\
\hline 6 PTSD-post-treatment & .25270 & .031732 & 10 \\
\hline Total & .24592 & .027303 & 60 \\
\hline
\end{tabular}

Levene's Test of Equality of Error

\section{Variances $^{\mathrm{a}}$}

Dependent Variable: Meanspeed Mean speed

\begin{tabular}{c|r|r|r}
$\mathrm{F}$ & $\mathrm{df1}$ & \multicolumn{1}{c|}{$\mathrm{df2}$} & \multicolumn{1}{c}{ Sig. } \\
\hline .662 & 5 & 54 & .654 \\
\hline
\end{tabular}

Tests the null hypothesis that the error

variance of the dependent variable is equal

across groups.

a. Design: Intercept + Group

Tests of Between-Subjects Effects

Dependent Variable: Meanspeed Mean speed

\begin{tabular}{|c|c|c|c|c|c|c|}
\hline Source & $\begin{array}{c}\text { Type III Sum } \\
\text { of Squares }\end{array}$ & df & Mean Square & $\mathrm{F}$ & Sig. & $\begin{array}{c}\text { Partial Eta } \\
\text { Squared }\end{array}$ \\
\hline Corrected Model & $.005^{\mathrm{a}}$ & 5 & .001 & 1.441 & .224 & .118 \\
\hline Intercept & 3.629 & 1 & 3.629 & 5049.467 & .000 & .989 \\
\hline Group & .005 & 5 & .001 & 1.441 & .224 & .118 \\
\hline Error & .039 & 54 & .001 & & & \\
\hline Total & 3.672 & 60 & & & & \\
\hline Corrected Total & .044 & 59 & & & & \\
\hline
\end{tabular}

a. R Squared $=.118$ (Adjusted R Squared $=.036$ ) 


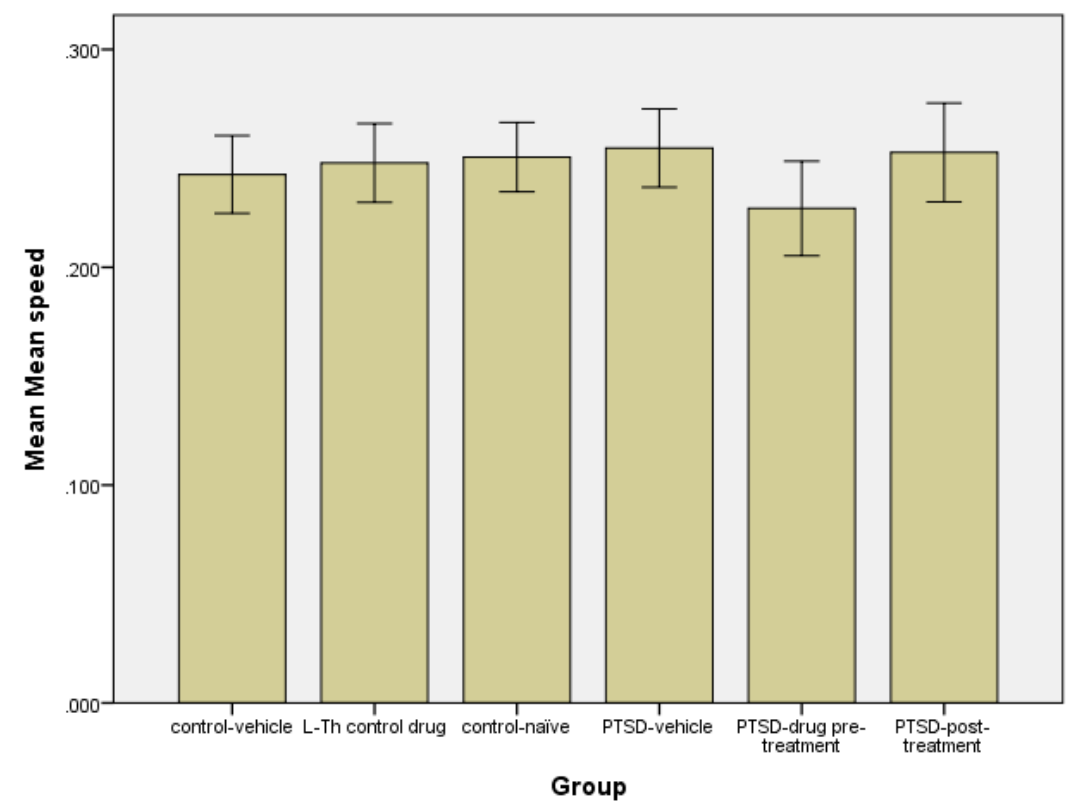

Error bars: $95 \% \mathrm{Cl}$

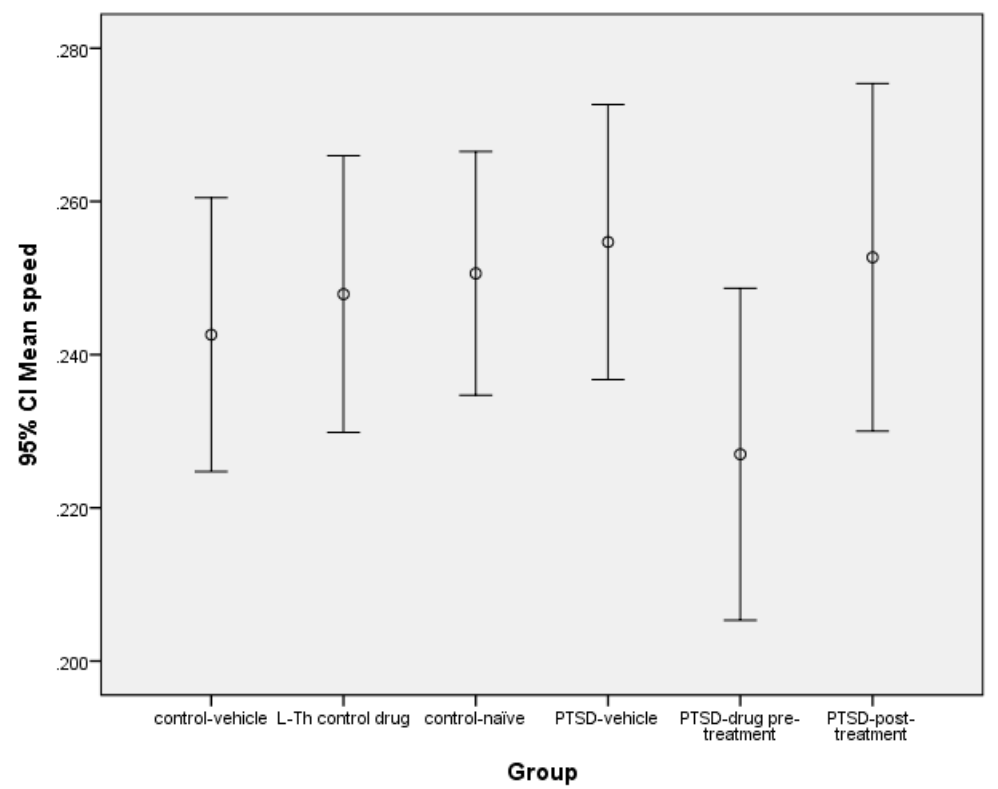

Summary: For the Mean Speed outcome, there was not a significant difference between the six groups: $F(5,54)=1.44, p=.224\left(\eta^{2}=.118\right)$. Though not significant the PTSD-vehicle group has the highest mean $(M=.2547)$ and PTSD-drug pre-treatment the lowest $(M=.227)$. 


\section{Max Speed}

Descriptive Statistics

Dependent Variable: Maxspeed Max speed

\begin{tabular}{ll|r|r} 
Group & Mean & Std. Deviation & \multicolumn{1}{l}{ N } \\
\hline 1 control-vehicle & .41310 & .027522 & 10 \\
\hline 2 L-Th control drug & .41430 & .021623 & 10 \\
\hline 3 control-naïve & .41240 & .037648 & 10 \\
\hline 4 PTSD-vehicle & .39990 & .032597 & 10 \\
\hline $\begin{array}{l}\text { 5 PTSD-drug pre- } \\
\text { treatment }\end{array}$ & .39510 & .019128 & 10 \\
\hline 6 PTSD-post-treatment & .39020 & .049502 & 10 \\
\hline Total & .40417 & .032974 & 60 \\
\hline
\end{tabular}

\section{Levene's Test of Equality of Error Variances $^{\mathrm{a}}$}

Dependent Variable: Maxspeed Max speed

\begin{tabular}{l|r|r|r}
$\mathrm{F}$ & $\mathrm{df1}$ & \multicolumn{1}{c|}{$\mathrm{df2}$} & \multicolumn{1}{l}{ Sig. } \\
\hline 1.917 & 5 & 54 & .107 \\
\hline
\end{tabular}

Tests the null hypothesis that the error variance of the dependent variable is equal across groups.

a. Design: Intercept + Group

Tests of Between-Subjects Effects

Dependent Variable: Maxspeed Max speed

\begin{tabular}{|c|c|c|c|c|c|c|}
\hline Source & $\begin{array}{l}\text { Type III Sum } \\
\text { of Squares }\end{array}$ & df & Mean Square & $\mathrm{F}$ & Sig. & $\begin{array}{l}\text { Partial Eta } \\
\text { Squared }\end{array}$ \\
\hline Corrected Model & $.005^{\mathrm{a}}$ & 5 & .001 & 1.004 & .424 & .085 \\
\hline Intercept & 9.801 & 1 & 9.801 & 9017.704 & .000 & .994 \\
\hline Group & .005 & 5 & .001 & 1.004 & .424 & .085 \\
\hline Error & .059 & 54 & .001 & & & \\
\hline Total & 9.865 & 60 & & & & \\
\hline Corrected Total & .064 & 59 & & & & \\
\hline
\end{tabular}

a. R Squared $=.085$ (Adjusted R Squared $=.000$ ) 

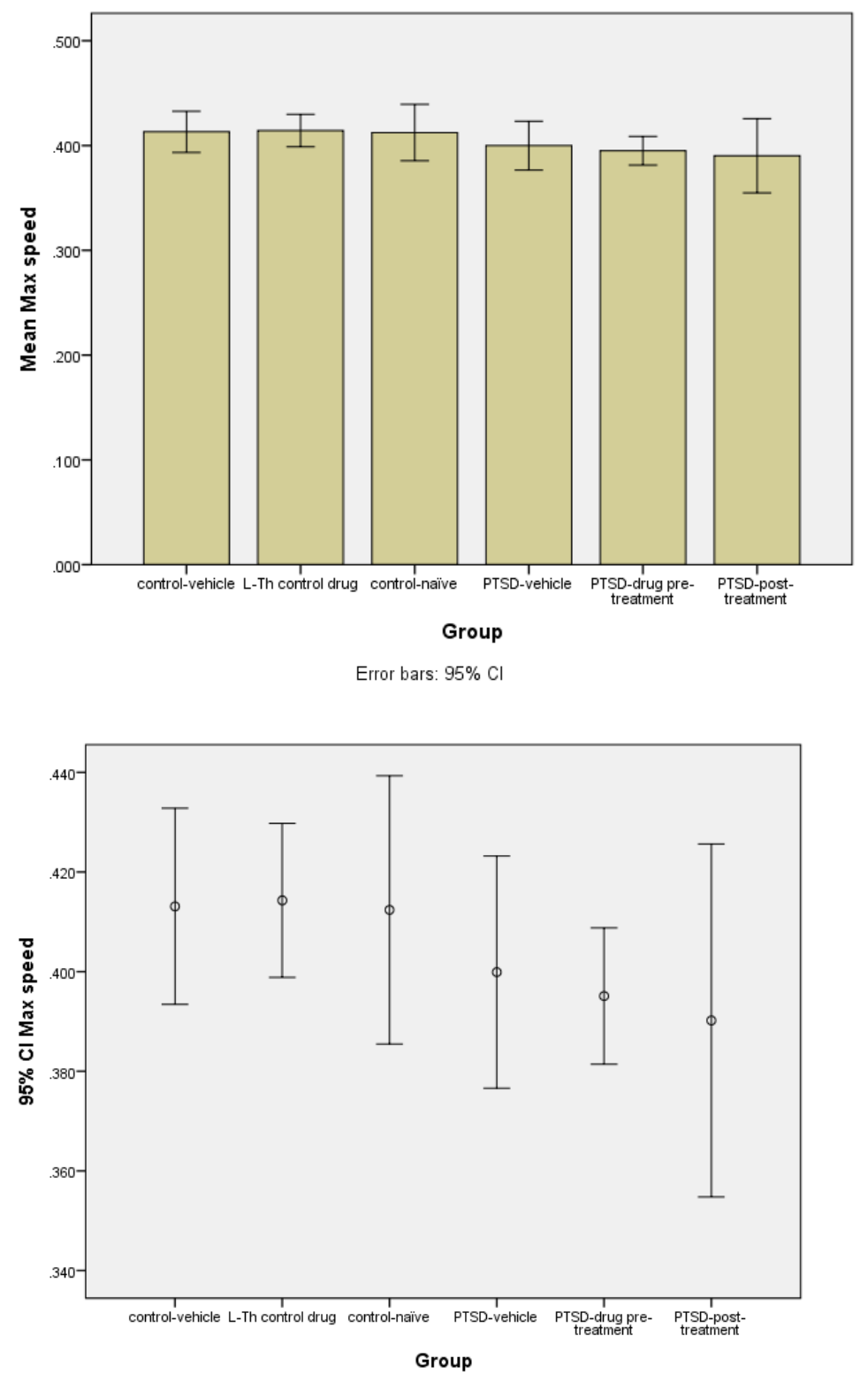

Summary: For the Max Speed outcome, there was not a significant difference between the six groups: $F(5,54)=1.00, p=.424\left(\eta^{2}=.085\right)$. Though not significant the L-Th control group has the highest mean $(M=.414)$ and PTSD-post-treatment the lowest $(M=.39)$. 


\section{Zone 1 time}

\section{Descriptive Statistics}

Dependent Variable: ZONE1 time ZONE 1 : time

\begin{tabular}{ll|r|r} 
Group & Mean & Std. Deviation & \multicolumn{1}{l}{ N } \\
\hline 1 control-vehicle & 14.460 & 5.8523 & 10 \\
\hline 2 L-Th control drug & 15.200 & 4.7754 & 10 \\
\hline 3 control-naïve & 19.370 & 4.8383 & 10 \\
\hline 4 PTSD-vehicle & 17.090 & 5.5065 & 10 \\
\hline $\begin{array}{l}\text { 5 PTSD-drug pre- } \\
\text { treatment }\end{array}$ & 19.180 & 8.7035 & 10 \\
\hline 6 PTSD-post-treatment & 16.360 & 5.4584 & 10 \\
\hline Total & 16.943 & 6.0388 & 60 \\
\hline
\end{tabular}

\section{Levene's Test of Equality of Error} Variances $^{a}$

Dependent Variable: ZONE1 time ZONE 1 : time

\begin{tabular}{l|r|r|l}
$\mathrm{F}$ & \multicolumn{1}{|c|}{$\mathrm{df1}$} & \multicolumn{1}{c|}{ df2 } & \multicolumn{1}{l}{ Sig. } \\
\hline 1.015 & 5 & 54 & .418 \\
\hline
\end{tabular}

Tests the null hypothesis that the error variance of the dependent variable is equal across groups.

a. Design: Intercept + Group

Tests of Between-Subjects Effects

Dependent Variable: ZONE1 time ZONE 1 : time

\begin{tabular}{l|r|r|r|r|r|r} 
Source & $\begin{array}{c}\text { Type III Sum } \\
\text { of Squares }\end{array}$ & df & Mean Square & \multicolumn{1}{c}{ F } & Sig. & \multicolumn{2}{c}{$\begin{array}{c}\text { Partial Eta } \\
\text { Squared }\end{array}$} \\
\hline Corrected Model & $204.593^{\text {a }}$ & 5 & 40.919 & 1.135 & .353 & .095 \\
\hline Intercept & 17224.593 & 1 & 17224.593 & 477.735 & .000 & .898 \\
\hline Group & 204.593 & 5 & 40.919 & 1.135 & .353 & .095 \\
\hline Error & 1946.954 & 54 & 36.055 & & & \\
\hline Total & 19376.140 & 60 & & & & \\
\hline Corrected Total & 2151.547 & 59 & & & & \\
\hline
\end{tabular}

a. $\mathrm{R}$ Squared $=.095$ (Adjusted R Squared $=.011$ ) 

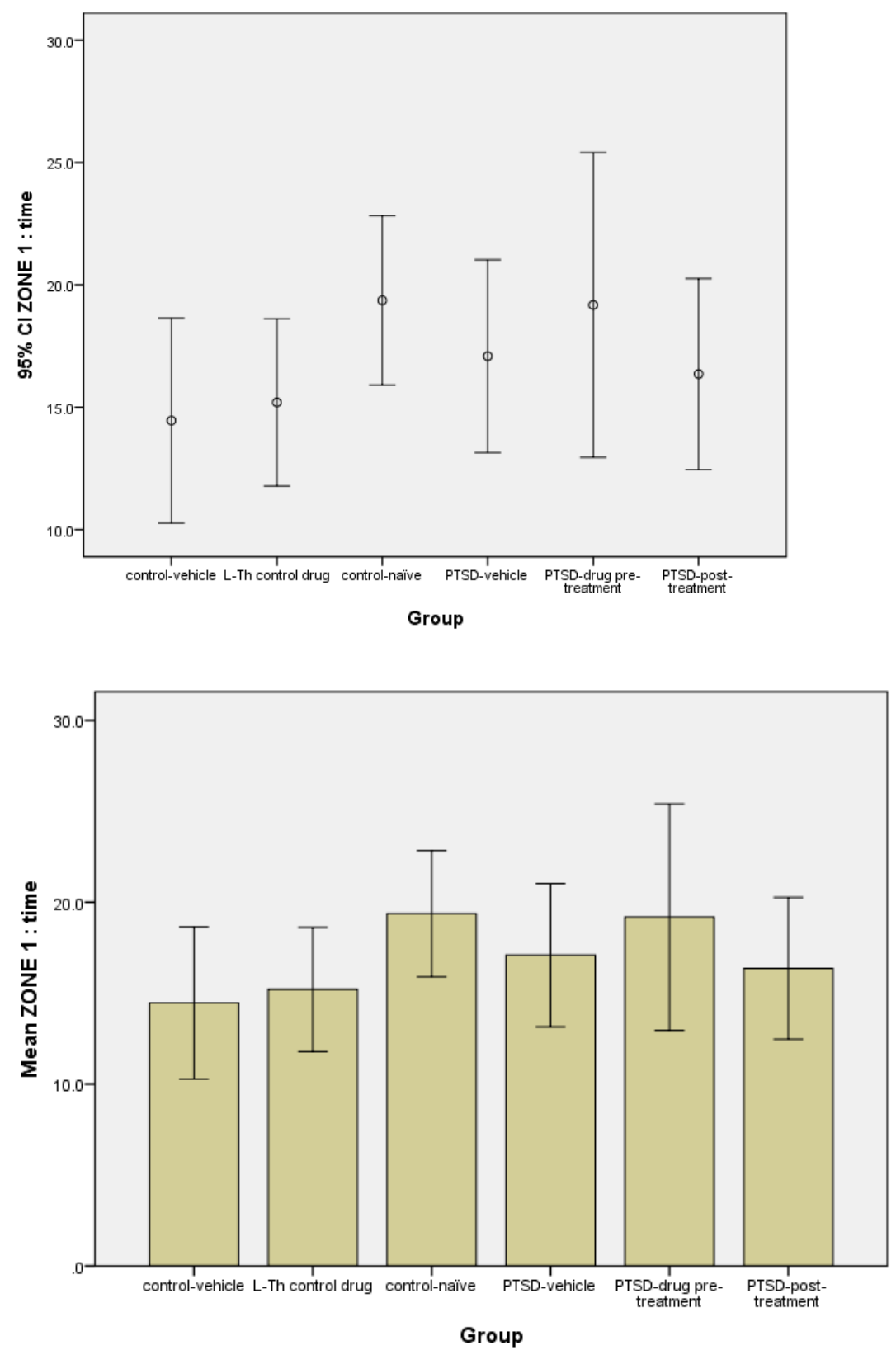

Error bars: $95 \% \mathrm{Cl}$

Summary: For the Zone 1 Time outcome, there was not a significant difference between the six groups: $F(5,54)=1.14, p=.353\left(\eta^{2}=.095\right)$. Though not significant the control naïve group has the highest mean $(M=19.37)$ and the control vehicle group the lowest $(M=14.46)$. 
Report

ZONE1time

\begin{tabular}{|l|r|r|r|r|}
\hline $\begin{array}{l}\text { Grou } \\
\mathrm{p}\end{array}$ & Mean & $\mathrm{N}$ & \multicolumn{1}{c|}{$\begin{array}{c}\text { Std. } \\
\text { Deviation }\end{array}$} & $\begin{array}{c}\text { Std. Error } \\
\text { of Mean }\end{array}$ \\
\hline 1 & 14.46 & 10 & 5.852 & 1.851 \\
2 & 15.20 & 10 & 4.775 & 1.510 \\
3 & 19.37 & 10 & 4.838 & 1.530 \\
4 & 17.09 & 10 & 5.506 & 1.741 \\
5 & 19.18 & 10 & 8.703 & 2.752 \\
6 & 16.36 & 10 & 5.458 & 1.726 \\
Total & 16.94 & 60 & 6.039 & .780 \\
\hline
\end{tabular}

